\title{
Critical Currents of Ideal Quantum Hall Superfluids
}

\author{
M. Abolfath ${ }^{1}$, A. H. MacDonald ${ }^{1}$, and Leo Radzihovsky ${ }^{2}$ \\ ${ }^{1}$ Department of Physics, University of Texas at Austin, Austin TX 78712 \\ ${ }^{2}$ Department of Physics, University of Colorado, Boulder, CO 80309
}

(October 31, 2018)

\begin{abstract}
Filling factor $\nu=1$ bilayer electron systems in the quantum Hall regime have an excitoniccondensate superfluid ground state when the layer separation $d$ is less than a critical value $d_{c}$. On a quantum Hall plateau current injected and removed through one of the two layers drives a dissipationless edge current that carries parallel currents, and a dissipationless bulk supercurrent that carries opposing currents in the two layers. In this paper we discuss the theory of finite supercurrent bilayer states, both in the presence and in the absence of symmetry breaking inter-layer hybridization. Solutions to the microscopic mean-field equations exist at all condensate phase winding rates for zero and sufficiently weak hybridization strengths. We find, however, that collective instabilities occur when the supercurrent exceeds a critical value determined primarily by a competition between direct and exchange inter-layer Coulomb interactions. The critical current is estimated using a local stability criterion and varies as $\left(d_{c}-d\right)^{1 / 2}$ when $d$ approaches $d_{c}$ from below. For large inter-layer hybridization, we find that the critical current is limited by a soliton instability of microscopic origin.
\end{abstract}

PACS number: 73.40.Hm,73.20.Dx

\section{INTRODUCTION}

In bilayer quantum Hall systems, broken symmetry ground states $[1,2]$ that have spontaneous interlayer phase coherence were predicted some time ago [3-8]. This broken symmetry state is expected to be most robust near total Landau level filling factor $\nu=1$ and occurs only when interactions between electron in opposite layers are comparable in strength to interactions between electrons in the same layer. The putative existence of this broken symmetry was used several years ago to explain [9] the observation of a surprisingly strong dependence [10] of the bilayer system $\nu=1$ charged excitation gap on in-plane magnetic field strength.

Spontaneous coherence between electrons in different energy bands is an old topic in condensed matter physics, although it has not yet been convincingly demonstrated outside of the quantum Hall regime. For example, it has long [11] been realized that spontaneous coherence at zero magnetic field is a possibility when overlapping or nearby bands have opposite quasiparticle energy vs. wavevector curvatures, the conduction and valence bands in a semiconductor or a semimetal in particular. Recent studies of optically generated electron-hole plasmas in semiconductors, do indeed hint [12] at the expected collective behavior. In a separate materials system, the discovery of weak ferromagnetism in lightly-doped divalent hexaborides [13], which are ferromagnetic despite the absence of partially filled $d$ - or $f$ - orbitals, led Zhitomirsky et al. [14] to propose recently that spontaneous coherence between conduction and valence bands could be the mechanism responsible for their ferromagnetism. Spontaneous coherence between different hyperfine states in Bose-Einstein condensates of magnetically confined ${ }^{87} \mathrm{Rb}$ atoms, manifested by Rabi oscillations between the two- components, is also closely related to the quantum Hall bilayer phenomena studied here. [15]

Spontaneous coherence states are most commonly described using the language of semiconductor physics in which a particle-hole transformation is performed for the valence band; spontaneous coherence between the bands then maps to electron-hole pair condensation, something that is closely analogous to Cooper pair condensation in a superconductor. These ordered states can be equivalently described as a pseudo-spin- $1 / 2$ quantum ferromagnets with the two states (top or bottom layer label in a quantum Hall bilayer system, band-index in a bulk semiconductor, or alkali atom species label in an ultra-cold atom system) defining an ordered spinor [9]. The ordered state has superfluid properties for "staggered" currents that flow in opposite directions in the two bands, excitonic superfluidity in the language of semiconductors. It was suggested more than twenty years ago [16] that spontaneous coherence could occur between two-dimensional (2D) conduction and valence bands localized in separate quantum wells and that $[17,18]$, at least in mean-field theory, the conditions required for condensation were more likely to be met when the 2D systems experienced a strong perpendicular magnetic field. It has not always been recognized, however, that because of the dispersionless Landau bands that occur in a strong magnetic field, there is no difference [4] in this regime between spontaneous coherence between a conduction band and a valence bands and spontaneous coherence between two conduction bands. The spontaneous coherence that occurs in bilayer quantum Hall systems is, in fact, precisely that originally anticipated by early theoretical work. $[17,18]$. Advances in our understanding of quantum Hall physics have, however, given us a deeper appreciation of the limitations of the mean-field theory approach used in the 
older work and of the physics behind its partial success. Superflow of the electron-hole pair condensate is perhaps the most characteristic property of the bilayer quantum Hall broken symmetry states, which we refer to here as quantum Hall superfluids. In this paper we discuss predictions for the maximum sustainable (counter-flowing) staggered supercurrents supported by these states that follow from microscopic mean-field theory for the ideal case in which the two-dimensional electron layers are completely free from disorder.

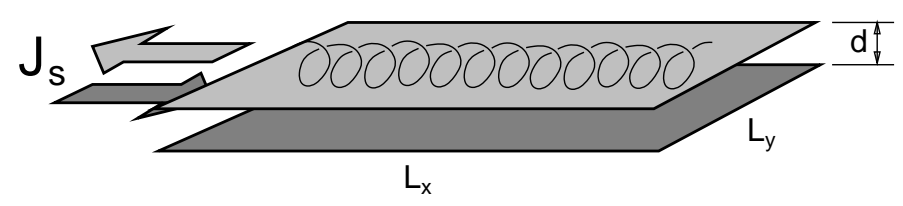

FIG. 1. A schematic illustration of a bilayer quantum Hall bar. For $\nu=1$ and sufficiently small interlayer spacing $d$ this system exhibits spontaneous interlayer phase coherence and excitonic superfluidity that supports counter-flowing (staggered) supercurrent $J_{s}$. The spiral is intended to indicate a uniform interlayer phase gradient that leads to constant $J_{s}$ along $x$.

The work presented here complements an earlier fieldtheoretic investigation by Kyriakidis and one us [19], who studied the decay of staggered supercurrents in bilayers quantum Hall superfluids by thermally activated phase slips and calculated the staggered I-V characteristics and critical current as function of interlayer gate voltage. The main technical difference between the two studies is that in the work of Ref. [19] it was assumed that the phase slip instability of a super-current carrying state is at long wavelengths, an assumption that turns out to be valid only for screened short-range interactions. As we explicitly show here, for realistic (long-range) Coulomb interactions, the instability is at a finite wavevector. Furthermore, we argue that even with short-range electronelectron interactions, when the interlayer spacing $d$ is near the critical value $d_{c}$ (determined by the transition out of interlayer coherent quantum Hall state), the instability is also at short microscopic scales of order the magnetic length. This finding limits the range of validity of the field theoretic approach of Ref. [19] to the case of very closely spaced layers, which cannot yet be realized experimentally.

Recently experimental advances by Eisenstein and collaborators [20-23] have revealed several dramatic effects that are only partially understood but are believed to be due to collective transport effects in quantum Hall superfluids. It appears, however, that the samples studied in current experiments have only short-range order because of disorder. Their progress, which we believe will open up a set of subtle new questions about non-equilibrium properties of superfluid-like ordered states, provides pow- erful motivation for the present work. The key capability which allows collective particle-hole transport effects to be probed by electrical experiments is the possibility of making separate electrical contact to two-dimensional electron gas layers with a separation $d \approx 20 \mathrm{~nm}$, small enough that is to be in the range where spontaneous coherence occurs. The strong zero-bias peaks they see in interlayer tunneling conductance [21] studies partially confirm predictions $[24,8,25]$ of Josephson-like effects, although these experiments are not fully understood and two of us has argued elsewhere that the analogy with the dc Josephson effect is incomplete. [26,27]. The evolution of the tunneling peak when the in-plane field [22] is varied shows evidence of the predicted $[3,7,25,28]$ linearly dispersing Goldstone collective mode associated with excitonic superfluidity. Most directly related to the present theoretical paper, is a very recent experiment [23], which studies transport properties of a quantum Hall superfluid for the case in which current (integrated across the sample) flows through only one of the two layers. Closely related transport phenomena have also been discussed theoretically [29] for the case of thin film ferromagnets.

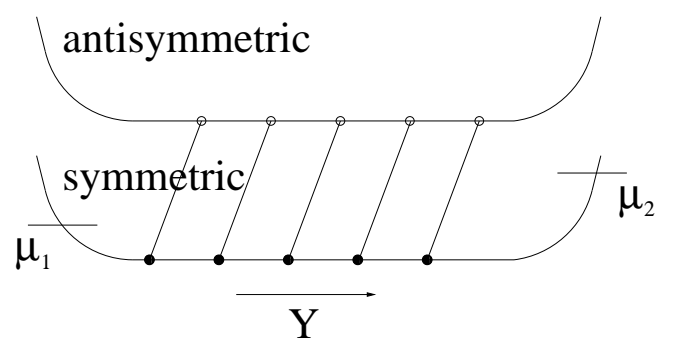

FIG. 2. Schematic non-equilibrium quasiparticle populations for the quantum Hall effect in a Hall bar geometry for an ideal disorder-free quantum Hall bilayer superfluid. The quasiparticle states are labeled by a guiding center coordinate which varies over the range from one edge of the sample to the other and is proportional to canonical momentum along the Hall bar. The quasiparticle current is equally divided between the two layers and a net current is maintained by having different chemical potentials on the two edges. The quasiparticles have spontaneous phase coherence and carry edge currents that are evenly partitioned between the two layers. In order to satisfy the drag experiment constraint that no net current to flow in one of the layers, the condensate must carry an electron-hole supercurrent equal in magnitude to the quasiparticle current. Unlike the charge current, the electron-hole supercurrent in the ideal case will flow uniformly through the bulk of the system. The mean-field state of a quantum Hall superfluid pairs electrons and holes that have different momenta in the current carrying direction, or equivalently, different cyclotron-motion guiding centers. This property is indicated schematically by the slanted lines that connect different guiding centers. 
The quantum Hall effect and superfluidity share the unusual property of transport without dissipation. In superfluids, dissipationless transport is possible because the quasiparticles are in equilibrium with a current-carrying condensate. In the quantum Hall effect, as illustrated schematically in Fig. 2, the quasiparticles are not in equilibrium. Instead the gap for charged excitations in the bulk implies that low-energy quasiparticles are localized at the sample edges, allowing a net current to be carried through the system [30] without dissipation by maintaining a Hall voltage difference between isolated subsystems on opposite edges. In the mean-field theory [31] of a quantum Hall superfluid, the occupied quasiparticle state wavefunctions are coherent linear combinations of orbitals localized in separate layers. For equal density in the bilayers, the current they carry is divided equally between the layers and any voltage probe that couples to the quasiparticle system will measure the same value in either layer, leading to large drag voltages [32]. Since the quasiparticles carry equal current in the two layers, the only way in which it is possible to have no net current in one of the layers, is to have it canceled by spontaneously generated staggered supercurrent, as illustrated in Fig. 3. In fact, as discussed in Ref. [33,34], since both staggered supercurrent and the uniform Hall current are dissipationless, at $\nu=1$ an ideal quantum Hall superfluid state should exhibit, respectively, vanishing and quantized longitudinal and Hall drag-resistivities.

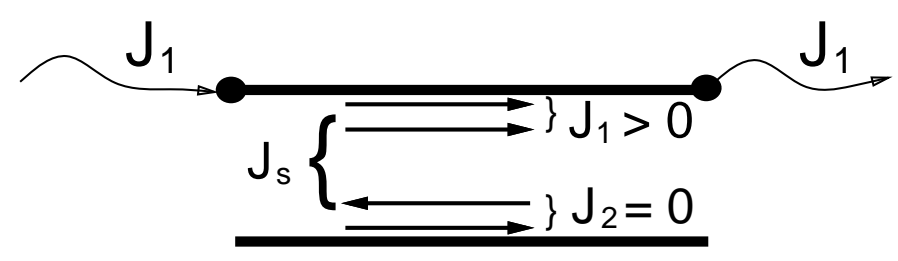

FIG. 3. Schematic for an integer quantum Hall superfluid bilayer in the drag geometry of Kellogg, et al., Ref.23, where electrical current $J_{1}$ is only allowed to flow in the top layer. To satisfy the boundary condition of vanishing current in the bottom layer, $J_{2}=0$, the system spontaneously develops a dissipationless staggered current $J_{s}$.

Current experiments are not fully in accord with this simple picture; the voltage drops in the current carrying direction are not zero and not identical in the two layers, and the Hall voltages are not perfectly quantized as one would expect in a $\nu=1$ quantum Hall bilayer superfluid at $T=0$. Most likely the discrepancy is due to disorder which leads [35] to mobile quasiparticle excitations in the bulk of the two-dimensional electron system and to vortex flow and consequent phase slips in the superfluid order parameter field. Long length scale inhomogeneity in the samples that leads to phase separation between ordered and disordered regions can also be invoked to explain [36] many aspects of current experimental results. The calculations we report in this paper do not account for disorder and do not attempt to explain quantitative aspects of current experiments. Instead we focus on the properties of ideal systems in which no disorder is present, with the expectation that the ideal situation will be approached more closely in the future. We expect the collective instability studied here to control longitudinal dissipation and deviation from Hall-drag quantization in clean quantum Hall bilayer superfluids.

In Section II of this paper we briefly summarize the mean-field-theory of current-carrying states in quantum Hall superfluids, allowing for the possibility of explicit symmetry breaking inter-layer hybridization terms in the microscopic Hamiltonian and for weak links in the twodimensional superfluid that are created by gate voltages. We define the critical current as the maximum current at which stable local minima in the energy-functional of the quantum Hall superfluid exist; formal expressions for the stability matrix at mean-field energy-functional extrema are also given in Section II. Some of these formal meanfield-theory results have been discussed in another context in earlier work, [37] but are included here for completeness. In Section III we apply these formal results to the case of a uniform quantum Hall superfluid with no inter-layer hybridization. In this case we are able to derive physically transparent expressions for the critical current. An important prediction that follows from this analysis is the way the critical current vanishes as the phase boundary that separates the quantum Hall superfluid from normal ground states of the 2D electron systems is approached. Section IV deals with the potentially interesting case [38] in which weak links are intentionally induced in these two-dimensional electron systems by applying voltages to external gates. We find that even relatively weak disturbances lead to drastically reduced critical currents. This partially explains the sensitivity of experimental samples to disorder, that will undoubtedly introduce such weak links in the superfluid bilayer. In this case we are able to derive a Josephson-like relationship between the order parameter phase jump across the weak link and the staggered supercurrent that flows across it.

An important difference between excitonic superfluids and both superconductors and fermion-pair superfluids is the inevitable presence in the Hamiltonian of explicit staggered gauge-symmetry breaking terms that allow electrons to tunnel between layers and hybridize quasiparticle states even when there are no interactions. These terms can be extremely weak but are never strictly zero. In Section $\mathrm{V}$ we show that staggered current causes a soliton lattice to form at a characteristic current density. For very strong tunneling, we find that the maximum supercurrent drops to zero. We conclude in Section VI with a summary of our results and a discussion of the role of quenched disorder and thermally activated vor- 
tices and phase slips that is informed by our numerical results for the microscopic properties of ideal systems. We conclude that non-linear transport in the drag experiment geometry used by Eisenstein and collaborators [23], studied as a function of carrier density, might be able to identify a crossover in dominant dissipation mechanism that is closely associated with the ideal system critical currents that are estimated in this paper.

\section{THE SUPERFLUID STATE ENERGY FUNCTIONAL: MEAN-FIELD EQUATIONS AND STABILITY ANALYSIS}

All of the calculations summarized in this article are based on a microscopic Hartree-Fock approximation (HFA) for the energy functional of quantum Hall staggered superfluid states and are analogous to ones made using the BCS theory for superconductors. We will consider only situations in which the order parameter is a function of a single $(X)$ spatial coordinate, making it convenient to choose a Landau gauge in which the Landau level orbitals are localized in this direction. Because we ignore edge effects, it will prove more convenient to choose orbitals localized along the direction of current $(\mathrm{X})$ flow rather than the more conventional choice perpendicular to the direction of current flow illustrated in Fig.2. The energy functional is $E_{M F}[\theta, \varphi]=$ $\left\langle\Psi\left|\hat{T}+V_{\text {ext }}+V_{e e}\right| \Psi\right\rangle$ where $T$ is the interlayer tunneling term, $V_{e x t}$ is the external potential term, and $V_{e e}$ is the Coulomb interaction in the microscopic Hamiltonian. The variational wavefunction used to define this energy functional is

$$
|\Psi\rangle=\prod_{X}\left(\cos [\theta(X) / 2] \hat{c}_{X T}^{\dagger}+\sin [\theta(X) / 2] e^{i \varphi(X)} \hat{c}_{X B}^{\dagger}\right)|0\rangle,
$$

where $X$ is a Landau gauge guiding center label, $T$ (top) and $B$ (bottom) are layer indices, and $\theta$ and $\varphi$ define the coherence factors of the BCS-like single-Slaterdeterminant many-particle wavefunction. If the phase coherent state is regarded as a pseudospin ferromagnet, the angles $\theta(X)$ and $\varphi(X)$ are polar and azimuthal angles that specify the direction of the pseudospin magnetization at position $X$. This is a special case of a class of variational wavefunctions in which virtual charge density fluctuations are not permitted. The energy functional can be written explicitly $[40,41,37]$ in terms of Hartree and exchange microscopic two-particle interaction matrix elements:

$$
\begin{gathered}
E_{M F}=-\frac{\Delta_{S A S}}{2} \sum_{X} \cos \varphi(X) \sin \theta(X)-\sum_{X} V_{g}(X) \cos \theta(X)+\frac{1}{2 L_{y}} \sum_{X, X^{\prime}}\left[H\left(X-X^{\prime}\right)-\frac{1}{2} F_{S}\left(X-X^{\prime}\right)\right] \cos \theta(X) \cos \theta\left(X^{\prime}\right) \\
-\frac{1}{4 L_{y}} \sum_{X, X^{\prime}} F_{D}\left(X-X^{\prime}\right) \sin \theta(X) \sin \theta\left(X^{\prime}\right) \cos \left[\varphi(X)-\varphi\left(X^{\prime}\right)\right]
\end{gathered}
$$

where $\Delta_{S A S}$ is the splitting between symmetric and antisymmetric orbitals induced by hybridization, $V_{g}(X)$ is the external gate voltage averaged over guiding center orbital $X$, and $H, F_{S}$, and $F_{D}$ are the Hartree matrix element, the same-layer exchange matrix element, and the different-layer exchange matrix element. If we neglect the finite widths of the quantum wells the interaction matrix elements are given by:

$$
\begin{aligned}
H(X) & =\int \frac{d q}{2 \pi} \frac{2 \pi e^{2}\left(1-e^{-q d}\right)}{2 \epsilon q} e^{i q X} e^{-q^{2} \ell^{2} / 2}, \\
F_{C}(X) & =e^{-X^{2} / 2 \ell^{2}} \int \frac{d q}{2 \pi} V_{C}\left(q, X / \ell^{2}\right) e^{-q^{2} \ell^{2} / 2}
\end{aligned}
$$

where $V_{C}\left(q_{x}, q_{y}\right)=2 \pi e^{2} /(\epsilon q)$ and $2 \pi e^{2} \exp (-q d) /(\epsilon q)$ for $C=S$ and $C=D$ respectively. Note that the exchange integral drops rapidly with orbit center separation, while the electrostatic integral falls only as $X^{-2}$ at large $X$, corresponding to the interaction between dipole moment lines created by charge transfer between the layers. In these equations $\ell \equiv \sqrt{\hbar c / e B}$ is the magnetic length. The Fourier transform of these functions will figure prominently in analyzing the superfluid properties of uniform bilayers. The energy functional $E_{M F}[\theta(X), \varphi(X)]$ can be elevated to a quantum theory by adding to it a Berry phase contribution to the Lagrangian $[9,39]$.
We choose the convention

$$
H(p)=\int \frac{d X}{2 \pi \ell^{2}} \exp (i p X) H(X)
$$

(and correspondingly for the exchange integrals) so that these quantities have units of energy. When finitethickness effects are neglected, we obtain the following explicit expressions for the Coulomb interaction case:

$$
\begin{aligned}
H(p) & =\frac{e^{2}}{2 \epsilon \ell^{2}} e^{-p^{2} \ell^{2} / 2} \frac{1-e^{-d|p|}}{|p|}, \\
F_{D}(p) & =\frac{e^{2}}{\epsilon} \int_{0}^{\infty} d r J_{0}\left(r p / \ell^{2}\right) e^{-r^{2} \ell^{2} / 2} e^{-r d},
\end{aligned}
$$


where $J_{0}(x)$ is the Bessel function. The same layer exchange function $F_{S}(p)$ differs only by the absence of the $e^{-r d}$ factor. $F_{D}(p)$ is plotted in Fig. 4 .

\section{A. Mean-field Equations}

Mean-field states of quantum Hall superfluids are extrema of the energy functional (2) and satisfy mean-field equations obtained by setting $\delta E_{M F} / \delta \varphi(X)=0$, and $\delta E_{M F} / \delta \theta(X)=0$. These Euler-Lagrange equations for the variables $\theta$ and $\varphi$ are coupled, and highly non-linear.
The equations are, in addition, strongly non-local because of the long range of the Coulomb interaction term. For $V_{g}(X)$ and $\Delta_{S A S}$ equal to zero, the global minimum of the energy functional is $\theta(X)=\pi / 2$ and $\varphi(X)$ equal to an arbitrary constant. Since we will be interested primarily in solutions with $\theta(X)$ near $\pi / 2$, it is useful to define $\eta(X)=\pi / 2-\theta(X)$. For our investigation we will be interested only in extrema that are periodic functions of $X$. Denoting the period by $a$ and using the thermodynamic limit property $\sum_{X} \rightarrow L_{y} \int d X /\left(2 \pi \ell^{2}\right)$, the mean-field equations can be written as:

$$
\begin{aligned}
\tan \varphi(X) & =\frac{\int_{0}^{a} \frac{d X^{\prime}}{2 \pi \ell^{2}} \cos \eta\left(X^{\prime}\right) \sin \varphi\left(X^{\prime}\right) \sum_{n=-\infty}^{\infty} F_{D}\left(X-X^{\prime}-n a\right)}{\Delta_{S A S}+\int_{0}^{a} \frac{d X^{\prime}}{2 \pi \ell^{2}} \cos \eta\left(X^{\prime}\right) \cos \varphi\left(X^{\prime}\right) \sum_{n=-\infty}^{\infty} F_{D}\left(X-X^{\prime}-n a\right)} \\
\tan \eta(X) & =\frac{2 V_{g}(X)+\int_{0}^{a} \frac{d X^{\prime}}{2 \pi \ell^{2}} \sin \eta\left(X^{\prime}\right) \sum_{n=-\infty}^{\infty}\left[F_{S}\left(X-X^{\prime}-n a\right)-2 H\left(X-X^{\prime}-n a\right)\right]}{\Delta_{S A S} \cos \varphi(X)+\int_{0}^{a} \frac{d X^{\prime}}{2 \pi \ell^{2}} \cos \eta\left(X^{\prime}\right) \cos \left[\varphi(X)-\varphi\left(X^{\prime}\right)\right] \sum_{n=-\infty}^{\infty} F_{D}\left(X-X^{\prime}-n a\right)}
\end{aligned}
$$

Our primary interest here is in current carrying states, and we choose $a$ to be the distance over which the condensate phase increases by $2 \pi$; our mean-field states are ones in which phase slips form a lattice. Note that $\sin (\varphi(X))$, $\cos (\varphi(X))$ and $\eta(X)$ are periodic. It follows that the total phase change of the order parameter on going from one end of the system to the other is $2 \pi L / a \equiv 2 \pi N_{w}$

These equations must be solved self-consistently to locate mean-field states. They can be understood most simply in the language of pseudo-ferromagnetism, in which they simply state that at each position $X$, the orientation of the quasiparticle pseudo-spinor is along the direction of the total pseudo-spin effective field, including direct and exchange mean-field interaction and external potential contributions felt by the quasiparticles at that point. The quasiparticles of the superfluid are in equilibrium with the condensate at extrema of the mean-field energy functional.

\section{B. General Expression for the Condensate Current}

The expression we use for the condensate staggered supercurrent follows from arguments presented in greater detail in previous work on quantum Hall superfluids [9] and implicitly in work on excitonic superfluidity. The current operator in each layer can be expressed in terms of the derivative of the Hamiltonian with respect to the vector potential in that layer. The vector potentials in the two layers can be varied by independent gauge transformations for the two layers and physical properties can depend on the phase difference between the two layers only through the gauge-invariant quantity $\hbar \nabla \varphi+(e / c)\left(\vec{A}_{T}-\vec{A}_{B}\right)$ where $\vec{A}_{T}$ and $\vec{A}_{B}$ are the vector potentials in top and bottom layers and $\varphi(X)$ is the dif- ference in wavefunction phase between top and bottom layers that appears in the HF variational wavefunction, Eq.1. Since the operator for the spatially averaged current in a layer is proportional to the derivative of the Hamiltonian with respect to the vector potential in that layer and we have chosen the same gauge for the vector potential for each layer, it follows that the spatially averaged condensate current is proportional to the rate of change of condensate energy with $N_{w}$ :

$$
J_{s}=\frac{1}{\pi \hbar} \frac{d E_{M F}}{L_{y} d N_{w}}=-\frac{a^{2}}{\pi \hbar} \frac{d \varepsilon_{M F}}{d a},
$$

where $\varepsilon_{M F}$ is the energy per cross-sectional area. This is the expression that we use below to extract condensate staggered currents from solutions of the mean-field equations. In Eq. $7 J_{s}$ is a number staggered current density, i.e. it corresponds to an electrical current density equal to $-e J_{s}(X)$ in the top layer and $e J_{s}(X)$ in the bottom layer. When tunneling between the layers is allowed, there is a circulating current within each period of the phase slip lattice in addition to this spatially averaged current. The circulating current is conveniently calculated from charge conservation which relates the current flowing between the layers to the divergence of the condensate supercurrent. The experimental meaning of the condensate current evaluated in this way is discussed at greater length in Section VI.

\section{Stability Analysis}

We restrict ourselves to the case of zero bias voltage for which the mean field solutions will have $\eta(X) \equiv 0$. A mean-field solution is a local minimum of the energy functional provided that the stability matrices 


$$
K_{\varphi \varphi}\left(X, X^{\prime}\right)=\frac{\delta^{2} E_{M F}}{\delta \varphi(X) \delta \varphi\left(X^{\prime}\right)}
$$

and

$$
\begin{aligned}
K_{z z}\left(X, X^{\prime}\right)= & \frac{\delta^{2} E_{M F}}{\delta \eta(X) \delta \eta\left(X^{\prime}\right)}, \quad(8 \mathrm{~b}) \\
K_{z z}\left(X, X^{\prime}\right)= & \frac{1}{2 L_{y}}\left[2 H\left(X-X^{\prime}\right)-F_{S}\left(X-X^{\prime}\right)\right] \\
& +\frac{1}{2} \delta_{X, X^{\prime}}\left[\Delta_{S A S} \cos (\varphi(X))+\frac{1}{L_{y}} \sum_{X^{\prime \prime}} F_{D}\left(X-X^{\prime \prime}\right) \cos \left(\varphi(X)-\varphi\left(X^{\prime \prime}\right)\right)\right] . \\
K_{\varphi \varphi}\left(X, X^{\prime}\right)= & -\frac{1}{2 L_{y}} F_{D}\left(X-X^{\prime}\right) \cos \left(\varphi(X)-\varphi\left(X^{\prime}\right)\right) \\
& +\frac{1}{2} \delta_{X, X^{\prime}}\left[\Delta_{S A S} \cos (\varphi(X))+\frac{1}{L_{y}} \sum_{X^{\prime \prime}} F_{D}\left(X-X^{\prime \prime}\right) \cos \left(\varphi(X)-\varphi\left(X^{\prime \prime}\right)\right)\right] .
\end{aligned}
$$

As we show in the next section, for $\Delta_{S A S}=0$, the mean-field solutions do not break translational invariance, and the stability matrices can be diagonalized explicitly by taking advantage of the translational symmetry property. The spectrum of the stability matrices can then be related to the Fourier transformed direct and exchange interaction matrix elements as we show below. In the more general case of finite $\Delta_{S A S}$, it is necessary to evaluate their spectrum numerically, which we do in Sec.V by discretizing the set of allowed guiding centers.

\section{CRITICAL CURRENT OF UNIFORM QUANTUM HALL SUPERFLUIDS $\left(\Delta_{S A S}=0\right)$}

For $\Delta_{S A S}=0$ and $V_{g}(X)$ equal to a constant, there are solutions to the mean-field equations for which $\eta$ (bilayer charge imbalance) is spatially uniform and $\varphi(X)$ varies at a constant rate. The largest possible supercurrents flow for balanced bilayers, i.e. for $V_{g}(X) \equiv 0$. The family of solutions with $\eta=0$ and $\varphi=Q X$ corresponds to states in which electron-hole pairs have condensed into a state with total momentum $Q \hat{x}$. Unlike the case of the zerofield fermion pair condensates, solutions to the mean field equations can be found for any pairing momentum $Q$, no matter how large. Inserting these solutions in Eq.(2), we find that the condensate-dependent part of the energy per unit area for pairing at wavevector $Q$ is $F_{D}(Q) / 8 \pi \ell^{2}$. The condensate current then follows from Eq.(7)

$$
J_{s}=\frac{2}{\hbar} \frac{d \varepsilon_{M F}}{d Q}=\frac{-1}{4 \pi \hbar \ell^{2}} \frac{2 d F_{D}(Q)}{d Q}
$$

The dependence of $J_{s}$ on pairing wavevector $Q$ is illustrated in Fig. 4 for a range of bias voltages. The natural unit of charge current density in the quantum Hall regime is $J_{0} \equiv 2 e^{3} / \hbar \epsilon \ell^{2} \approx 82 \mu \mathrm{A} / \mu \mathrm{m} B$ [Tesla].

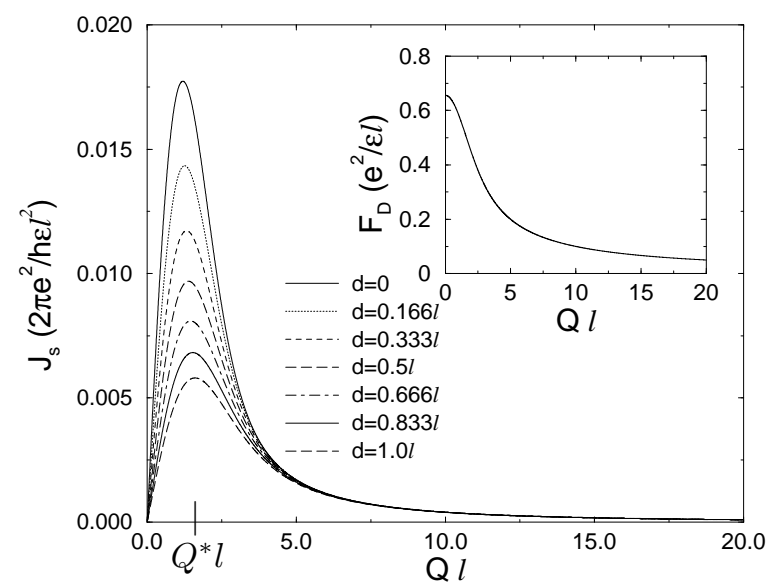

FIG. 4. $J_{s}$ vs. pairing wavevector $Q$ for $\Delta_{S A S}=V_{g}=0$ and a series of layer separation $d$ values. $J_{s}$ is in units of $J_{0} / e \equiv \frac{1}{\hbar} \frac{e^{2}}{\epsilon \ell^{2}}\left(J_{0}=41 \times 10^{-6} \mathrm{Amp} / \mu m B\right.$ [Tesla]. $)$ Our stability analysis demonstrates that the mean-field state is always unstable for pairing wavevectors larger than $Q^{*}$, the pairing wavevector at which $J_{s}$ is maximized. For quantum Hall superfluids, however, the critical current is almost always limited by unstable amplitude fluctuations of the electron-hole pair condensate that are indicated by negative values of $K_{z z}(q)$. For example for $d=\ell$, the maximum supercurrent mean-field state $\left(J_{c}=0.0058 J_{0}\right)$ occurs at $Q \ell=1.6$, but amplitude fluctuations are unstable for $Q \ell>1.2$ as we discuss below. The inset shows $F_{D}(Q)$ vs. $Q$ for $d=\ell$. $F_{D}(Q) \sim$ constant $-Q^{2}$ at small $Q$ and $\sim 1 / Q$ at large $Q$ so that $J_{s}$ is proportional to $Q$ and $Q^{-2}$ at large and small $Q$ respectively.

The main effect of a uniform bias voltage is to reduce the scale for supercurrent values. We should also remark that these supercurrent values and all numerical results discussed in this paper will be influenced somewhat by 
corrections that account for the finite thickness of the two-dimensional layers. These corrections could be incorporated into our calculations without any difficulty, but we choose to ignore them here mainly for the sake of simplicity. As we discuss later, other more fundamental and difficult issues arise when we attempt to compare this analysis with experiments in real systems.

The Hamiltonian $H_{f}$ for Gaussian fluctuations $\phi(X)=$ $\varphi-Q X$ and $m_{z}(X)$ around these uniform superfluid $\left(\varphi_{0}=Q X, m_{z}=0\right)$ mean-field states can be expressed in terms of the stability matrices discussed previously. In the uniform current case, $K_{\varphi \varphi}\left(X, X^{\prime}\right)$ and $K_{z z}\left(X, X^{\prime}\right)$ depend only on $X-X^{\prime}$ and the matrices can be diagonalized by Fourier transformations.

We find that

$$
\begin{aligned}
\frac{H_{f}\left[\varphi, m_{z}\right]}{L_{x} L_{y}}= & \frac{1}{2} \sum_{q} \varphi(-q) K_{\varphi \varphi}(q) \varphi(q) \\
& +\frac{1}{2} \sum_{q} m_{z}(-q) K_{z z}(q) m_{z}(q)
\end{aligned}
$$

where

$$
\begin{aligned}
& K_{\varphi \varphi}(q)=\frac{1}{2 \pi \ell^{2}}\left[F_{D}(Q)-\frac{F_{D}(q+Q)+F_{D}(q-Q)}{2}\right] \\
& K_{z z}(q)=\frac{1}{2 \pi \ell^{2}}\left[H(q)-\frac{1}{2} F_{S}(q)+\frac{1}{2} F_{D}(Q)\right] .
\end{aligned}
$$

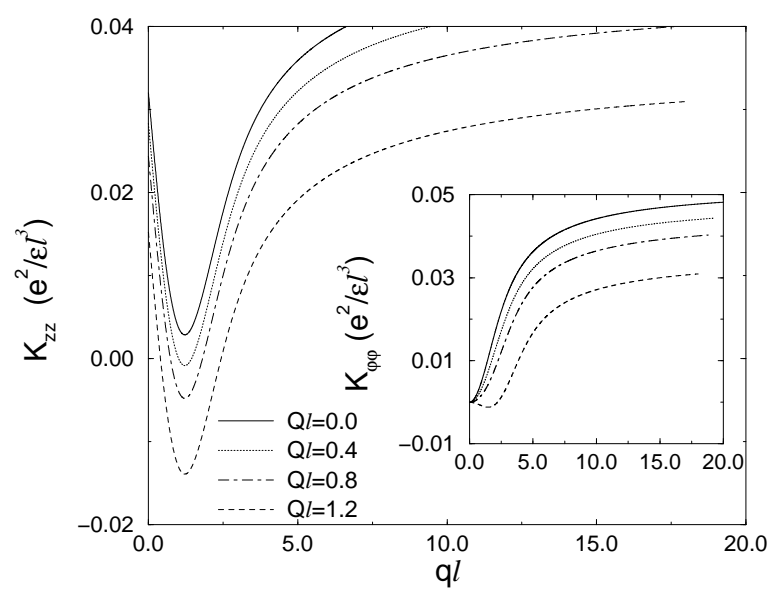

FIG. 5. $K_{z z}(q)$ and $K_{\varphi \varphi}(q)$ vs. $q$ for $d=\ell$, and $\Delta_{S A S}=0$. $K_{\varphi \varphi}(q=0)=0$ even at finite pairing momentum $Q \neq 0$, because the energy is invariant under a spatially constant change in the phase $\varphi . K_{z z}(q)$ is nonanalytic at $q=0$, decreasing in proportion to $|q| d$, because of long-range Coulomb interactions which favor non-uniform exciton density variations. The decrease of $K_{z z}(q)$ for $q d$ small is rapid for large layer separations $d$, and eventually drives the critical current to zero, destabilizing the superfluid state.

(In these equations we have used the following conventions for the Fourier transforms of $\phi(X)$ and
$m_{z}(X) . \varphi(q) \equiv \sum_{X} \phi(X) \exp (-i p X) / N_{\phi}$ where $N_{\phi}=$ $L_{x} L_{y} /\left(2 \pi \ell^{2}\right)$ is the total number of Landau gauge orbitals in the system. This choice is made so that $\varphi(q)$ is dimensionless and $\varphi(q=0)$ is the spatial average of $\phi(X)$.) The first two terms in Eq.(13b) represent the cost in electrostatic energy and the gain in exchange energy that accompanies fluctuations in the bilayer charge balance. Both stability kernels $K_{\varphi \varphi}$ and $K_{z z}$ must be positive definite for the superfluid state to be stable against small fluctuations. From Eq.(13b), we see that for $q \rightarrow 0$, $K_{\varphi \varphi} \rightarrow 4 \hbar q^{2} \frac{\partial J_{s}}{\partial Q}$, which is positive only on the increasing portion of the $J_{s}(Q)$ curve; at larger pairing wavevectors phase separation into regimes with larger and smaller average phase winding rates is always energetically preferred over the homogeneous state.

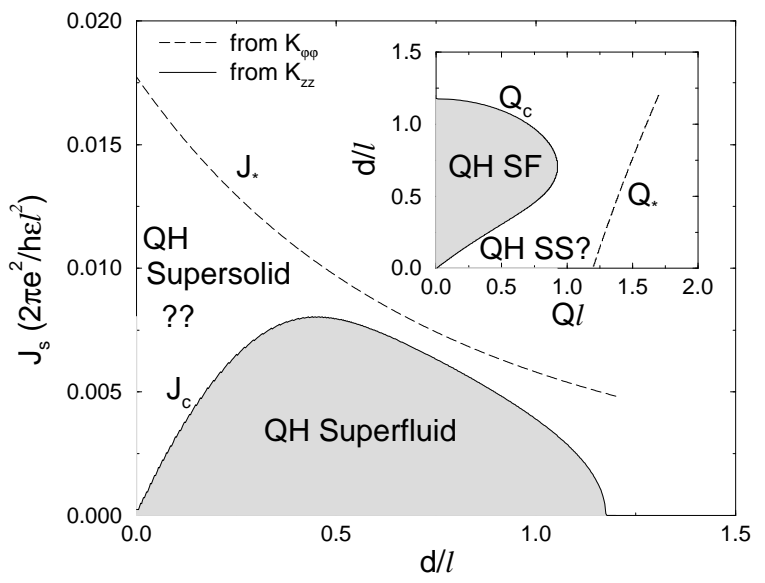

FIG. 6. Critical supercurrent density $J_{c}(d)$ (main panel) and (inset) pairing wavevector $Q_{c}(d)$ of the critical current vs. layer separation $d$, defining the $\mathrm{QH}$ Superfluid phase boundary in pairing wavevector-layer separation space. The dashed lines in the main figure and the inset showing critical current, $J_{*}$ and maximum pairing wavevectors, $Q_{*}$ obtained by applying the $K_{\varphi \varphi}$ (condensate phase) order parameter stability criterion is shown by these results to be less stringent; the solid line is determined by the $K_{z z}$ stability criterion. For $d \approx d_{c}, J_{c} \propto\left(d_{c}-d\right)^{1 / 2}$, while for $d \rightarrow 0 J_{c} \propto d$. Current experimental samples have layer separations close to the critical value $d_{c}$. Because the $K_{z z}(q)$ instability is at a finite wavevector $q_{*}$, we expect that for small $d$ the resulting current carrying state is a modulated $\mathrm{QH}$ superfluid, i.e., a QH supersolid. For larger values of $d$, the instability is at a large finite wavevector and we expect a first order transition between uniform superfluid and disordered ground states.

For $q \rightarrow 0$ and $Q=0, K_{\varphi \varphi} \rightarrow \rho_{s} q^{2}$ where $\rho_{s}$ is phase stiffness (superfluid density) parameter of the superfluid. $K_{\varphi \varphi}(q)$ appears to always be positive when it is positive for $q \rightarrow 0$; the $K_{\varphi \varphi}$ stability criterion $Q<Q^{*}$ (with $Q^{*}$ the pairing wavevector at which $J_{s}(Q)$ is maximum) does not limit the critical current of quantum Hall superfluid to values below the maximum value at $Q^{*}$ that 
occurs in mean-field solutions. In contrast, $K_{z z}(q)$ always has its minimum at a finite value of $q$. This differs from the field-theoretic treatment of Ref. [19], where $K_{z z}(q)$ was taken to be a monotonically increasing function of $q$. This assumption is not valid for unscreened (long-range) Coulomb interactions. The last term in the expression for $K_{z z}(q)$, which represents the loss in condensation energy when the condensate electron-hole density is varied from its optimal value, is essential for the stability of quantum Hall superfluids at typical values of $d / \ell$. As illustrated in the inset of Fig.4, this stabilizing term decreases in magnitude at finite $Q$ because of the reduced condensation energy associated with finite $Q$ pairing. It is this reduction in condensation energy that limits the critical staggered supercurrent in our theory. It is also worth remarking that $K_{z z}(q)$ is negative for any finite value of $Q$ for $d=0$. It is only at $d=0$ that the ground state is given exactly by the Halperin [1] $(1,1,1)$ two-component quantum Hall fluid wavefunction. It follows that the $(1,1,1)$ state is not a superfluid, a point that we have made [42] previously. Because of this property, field-theoretical analyses of quantum Hall superfluid properties that start from $(1,1,1)$ variational wavefunctions should be regarded with some caution, in our view.

In Fig. 6 we have plotted the layer separation dependence of the ideal bilayer critical current $J_{c}$ that follows from this stability analysis. The critical current vanishes as $d \rightarrow 0$ because of the vanishing difference between intralayer and interlayer interactions that is necessary to provide a barrier to phase slip nucleation. In the language of pseudo-ferromagnetism $[9,50]$, the corresponding observation is that easy-plane anisotropy is required for spiral state metastability. The staggered critical supercurrent also vanishes at large layer separation because the stiffness against electron-hole (bilayer charge imbalance) density waves vanishes as we discuss below. This figure shows that the critical current is always limited by the $K_{z z}(q)$ stability criterion. The maximum critical current occurs for $d \sim 0.4 \ell$ and is $\sim 0.008$ of our currentdensity unit $J_{0}$. It follows from this analysis that under typical experimental circumstances the critical current of an ideal system should be $\sim 0.33 \mu \mathrm{A} \mu \mathrm{m}^{-1}$. This staggered critical current value is several orders of magnitude larger than the value used in recent drag experiments [23]. Although existing drag experiments provide evidence of collective transport, they explicitly demonstrate dissipation in the pseudospin current channel because the measured longitudinal electric fields in the two layers are different. This dissipation is likely due to the flow of vortices induced by thermal fluctuations and disorder that we do not account for here. It would nevertheless be interesting to experimentally explore the possibility that a large increase in the difference in longitudinal electric fields would occur relatively sharply at larger current densities, reflecting a change in the dominant dissipation mechanism when the ideal critical current addressed in this paper is exceeded.

The $K_{\varphi \varphi}(q)$, and $K_{z z}(q)$ stiffnesses in the fluctuation Hamiltonian are inversely related to static response functions. Since the phase of the condensate and the electron-hole pair density are canonically conjugate coordinates these two stiffnesses are also simply related to the system's quantized collective excitation energies. The semiclassical picture of these modes emerges from the Landau-Lifshits equations of motion of the pseudospin ferromagnet, which can be derived by adding a Berry phase term to the above fluctuation Hamiltonian to obtain the system's Lagrangian [9].

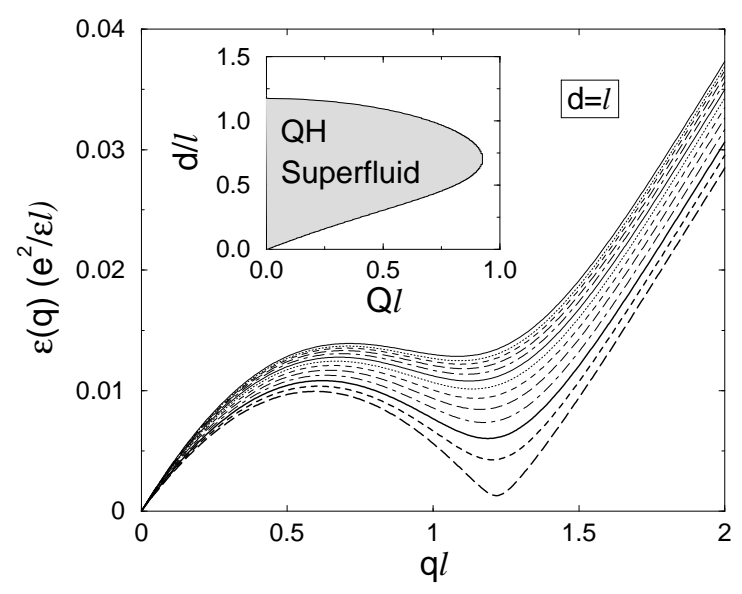

FIG. 7. The dispersion relation of the quantum Hall superfluid collective modes is plotted for $d=\ell$, and $V_{g}=\Delta_{S A S}=0$ for values of the pairing wavevector $Q$ which vary between $Q=0$ (the top curve) and $Q=0.7 \ell^{-1}$. At $Q=Q_{c}$, where the roton minima vanishes, the superfluid mean-field state is unstable.

The superfluid collective modes have energy $\varepsilon(q)=$ $2 \pi \ell^{2} \sqrt{K_{z z}(q) K_{\varphi \varphi}(q)}, \quad$ generalizing earlier results $[18,3,28,9]$ to the case of a finite supercurrent state. For all pairing wavevectors, the collective modes have a linear dispersion of a superfluid at long wavelengths. At shorter length scales (large $q) \varepsilon(q)$ also has a minimum, that is reminiscent of the roton minimum in superfluid Helium. The source of this characteristic minimum in the collective mode dispersion $\varepsilon(q)$ is the Coulomb interaction, which leads to the minimum in $K_{z z}(q)$ displayed in Fig.5. The collective mode dispersion is altered when a supercurrent is flowing primarily because of the reduction in the condensation energy and $K_{z z}(q)$ with increasing $Q$. When the minimum is already close to zero for $Q=0$, the critical supercurrent is small. Since $F_{D}(Q)$ is a quadratic function of $Q$ for small $Q$ and the minimum in $K_{z z}(q, Q=0)$ approaches zero linearly as $d \rightarrow d_{c}$, it follows that $Q_{c}$ and the critical staggered supercurrent vanish like $\left(d_{c}-d\right)^{1 / 2}$ for $d \rightarrow d_{c}$. 
The physics that limits the ideal critical current of quantum Hall superfluids is similar to that encapsulated by Landau's expression for the critical current of superfluid Helium, in which he argued that the superfluid velocity cannot exceed $J_{s}^{\max }=v_{s}^{\max } n=\min \left(\varepsilon^{*}(q) / \hbar q\right) n$, where $\varepsilon^{*}(q)$ is the elementary excitation dispersion relation when no supercurrent is flowing, i.e. at $Q=0$, and the minimum above is over all wavevectors $q$. In both superfluid Helium and quantum Hall superfluid cases, Landau's argument approximates the more fundamental requirement that the order parameter of the current-carrying state be at a local minimum of the model's energy functional. The remark that we make here applies mutatis mutandis to conventional superfluids. We first note that for small $Q, K_{z z}(q, Q) \approx$ $K_{z z}(q, 0)-\rho_{s} Q^{2}$ so that the maximum value of $Q$ is $\left[\min \left(K_{z z}(q, 0)\right) / \rho_{s}\right]^{1 / 2}$. One additional approximation is required to obtain the same result from the Landau criterion; we must assume that $K_{\varphi \varphi}(q, Q=0) \approx \rho_{s} q^{2}$ for $q$ up to the value at which $\varepsilon^{*}(q) / q$ is minimized, actually a reasonable approximation for quantum Hall superfluids. Using $n=\left(2 \pi \ell^{2}\right)^{-1}$, the density of a full Landau level, we see that the superfluid velocity is related to $Q$ by $v_{s}=\rho_{s} Q\left(2 \pi \ell^{2}\right) / \hbar$. The Landau criterion $v_{s}^{2}<\min \left[\varepsilon^{*}(q)^{2} / q^{2}\right] \approx\left(2 \pi \ell^{2}\right)^{2} \min \left[K_{z z}(q, 0) \rho_{s}\right]$ then leads to the same limit on $Q$ as the $K_{z z}(q, Q)>0$ stability criterion, that we use here to obtain the QH superfluid phase boundary illustrated in Figs.6,7.

\section{WEAK LINKS IN QUANTUM HALL SUPERFLUIDS $\left(\Delta_{S A S}=0\right)$}

One element of the physics of quantum Hall superfluids that might potentially be interesting for future experimental study, is the possibility [38] of creating and tuning weak links using gate voltages. Weak links are locations in the superfluid at which the local superfluid stiffness is reduced and phase changes are therefore concentrated in a narrow region (link). In quantum Hall bilayer superfluids, one such weak-link geometry is illustrated in Fig.8, where the local stiffness is reduced in situ by a gate voltage that locally moves the bilayer system away from balanced condition of equal density in the two layers.

In this section we discuss the supercurrent properties of quantum Hall superfluids with such top gate-voltage induced weak links. We represent the electrostatic potential from a narrow gate separated from the epitaxially grown bilayer system by a cap layer (Fig.8), somewhat crudely, by an external potential that has a narrow Gaussian variation in the $x$ direction, but is invariant in the $y$ direction, as shown in the inset of Fig.9. The width of the Gaussian should be given approximately by the minimum of the gate width and the thickness of the cap layer. [47]

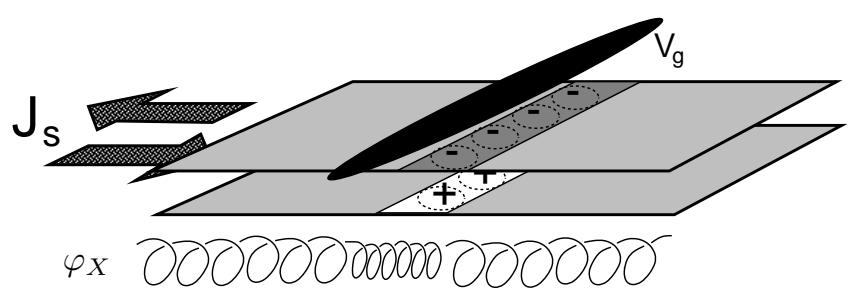

FIG. 8. Schematic illustration of a quantum Hall superfluid weak-link induced by a top gate. The pseudospin stiffness is reduced locally by a gate voltage that displaces the bilayer system away from the balanced condition of equal density in the two layers. The increased phase winding rate at the link, required by current conservation, is illustrated.

In order to evaluate the Josephson-like relationship between the phase change across a weak link and the superfluid current, we have solved the mean-field equations for a periodic system system with one weak-link and a $2 \pi$ phase change per period $a$. In this way we can vary the superfluid current by changing the period $a$. A typical solution is shown in Fig.9.

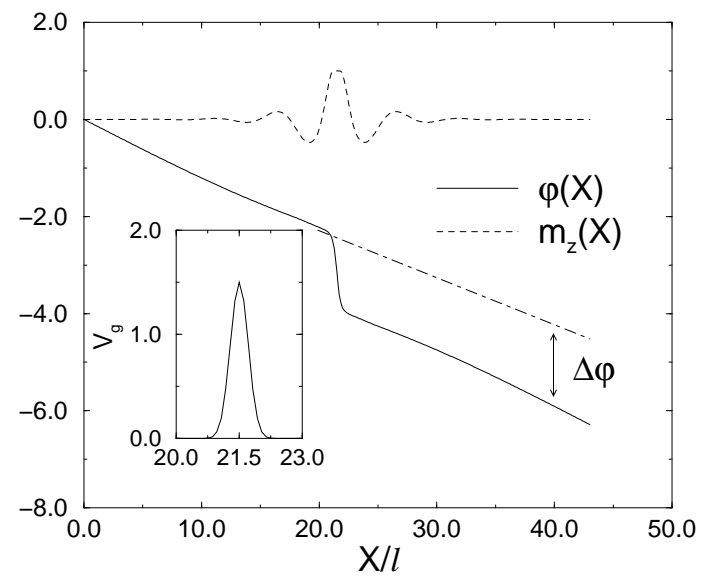

FIG. 9. $\varphi(X)$ and $m_{z}(X)$ are shown for a bilayer with $\Delta_{S A S}=0$ and $d=\ell$. These results are for a weak link potential with a width equal to $\ell$ and a barrier height $1.5 e^{2} / \epsilon \ell$. $\Delta \varphi$ is measured with respect to its value at one edge of the periodic cell and the weak link is located at the center of the well.

Since for vanishing interlayer tunneling, $\Delta_{S A S}=0$, considered here the dc staggered supercurrent is spatially uniform, current continuity demands $\partial \varphi / \partial X$ to be inversely related to the local superfluid density when the phase gradient is small. In particular, $\partial \varphi / \partial X$ must be constant to an excellent approximation away from the weak link. We will refer to this constant as $Q$ below. After solving the mean-field equations for a series of $Q$ 
values, the current density can be evaluated either by exploiting its relationship to the $a$ dependence of the total energy or by combining the phase winding rate $Q$ away from the weak link with the uniform system $J_{s}(Q)$ expression. The phase change across the weak link, $\Delta \varphi$, is extracted by extrapolating the constant curve from one side of the weak link to the other and finding the additional shift that is present because of the weak link, as illustrated Fig.9.

A typical result for $J_{s}$ vs. $\Delta \varphi$ obtained in this way is summarized in Fig.10.

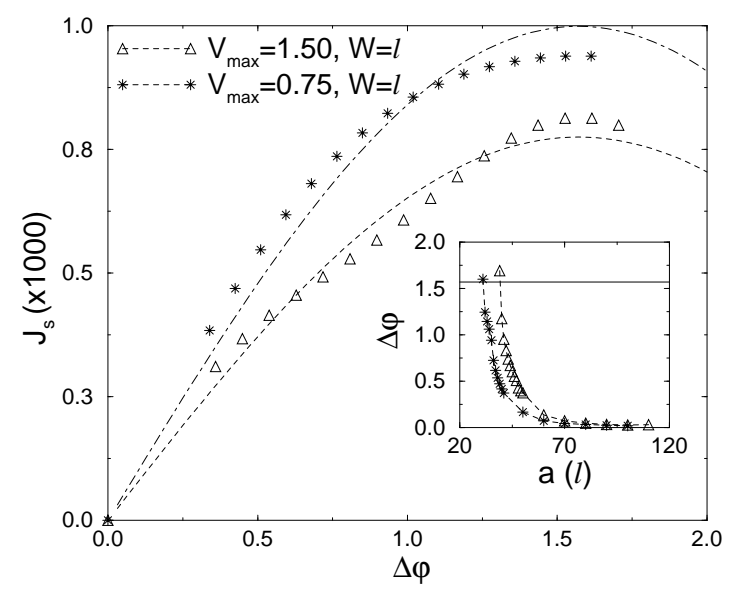

FIG. 10. Supercurrent density vs. weak-link phase slip angle $\Delta \varphi$ for weak links of width $\ell$ and heights $V_{g}^{\max }=0.75$ and $V_{g}^{\max }=1.50 e^{2} / \epsilon \ell$. The maximum supercurrents in this case are approximately five times less than in the absence of a weak link.

For small currents (large $a), J_{s}$ increases linearly with $\Delta \varphi$, as in a conventional Josephson junction. Notice that $J_{s}$ reaches its maximum for $\Delta \varphi$ close (but not exactly equal) to $\pi / 2$ as in the Ambegaokar-Baratoff [44] theory of superconductors linked by a tunneling Hamiltonian. The microscopic physics of the coupling is quite different in the quantum Hall superfluid, however. For a weak link that is translationally invariant along $y$ [47] (the geometry illustrated in Fig.8) the guiding center $X$ remains a good quantum number in the absence of disorder and consequently the quasiparticles do not tunnel across [47]. Instead, the coupling between the uniform superfluids on opposite sides of such weak link is due to Coulomb exchange interactions, both within and near to the weak link. As in the case of a uniform superfluid, configurations of the order parameter field that occur beyond the maximum of this function are not stable, and the staggered critical current is given by the maxima of these curves.

In Fig. 11 we plot critical currents as a function of the strength of the bias potential that produces the weak link for two different values of the width of the link. The crit- ical current decreases rapidly with both the width of the barrier potential and its strength. Because sample inhomogeneities will undoubtedly introduce a distribution of weak links into the superfluid bilayer, one conclusion that follows immediately from these calculations is that the critical current will likely sharply reduced by disorder in typical current samples.

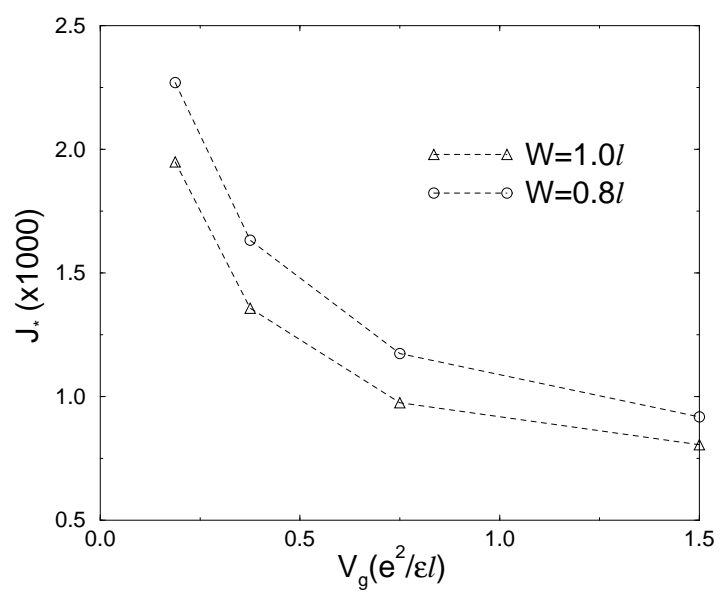

FIG. 11. Critical staggered supercurrent density dependence on the maximum of the bias potential $V_{g}^{\text {max }}$ responsible for the weak link, plotted for two width values.

We close this section by noting that to obtain the weaklink critical current $J_{c}$, we have relied solely on the vanishing $K_{\varphi \varphi}$ criterion, and have ignored the $K_{z z}$ stability matrix. Although we have not checked this explicitly, because a weak link substantially reduces the phase stiffness, leading to a five-fold reduction in $J_{*}$, we believe it is this $K_{\varphi \varphi}$ criterion that determines the weak-link critical current, giving $J_{c}=J_{*}$.

\section{ROLE OF INTERBAND HYBRIDIZATION IN QUANTUM HALL SUPERFLUIDS: THE $\Delta_{S A S} \neq 0$ CASE}

In the presence of interlayer tunneling $\Delta_{S A S}$, the superfluid energy functional has soliton lattice extrema which break translational symmetry but, as we comment later, still carry a finite supercurrent. It is not in general possible to address the metastability of these solutions analytically, although a qualitative understanding can be achieved using scaling arguments [19].

\section{A. $K_{\varphi \varphi}$ instabilities of charge-balanced bilayers}

To find members of this family of energy functional extrema, we first solve the mean-field equations selfconsistently to find $\varphi(X)$ solutions at $\eta(X) \equiv 0$. We 
seek solutions with period $a$, where $a$ is the distance between the soliton centers that are located at the midpoints of our primitive cells. For weak tunneling the solitons are wide and are accurately [40] approximated by solving the sine-Gordon equation that is obtained by minimizing the gradient approximation to the $\eta \equiv 0$ energy functional. It follows that for dilute solitons at small $\Delta_{S A S}, \varphi(X) \simeq 4 \tan ^{-1} \exp [-(X-a / 2) / \xi]$ modulo $2 \pi$ within each cell, where $\xi=\ell \sqrt{4 \pi \rho_{s} / \Delta_{S A S}}$ is the width of the soliton. We find numerically that as the density of solitons increases (the unit cell period $a$ decreases) and the energy per cross-sectional length increases, we eventually reach a situation at $a=a_{c}$ for which the soliton lattice solution is no longer metastable and the self-consistency procedure does not converge. This point is most conveniently identified numerically by reducing $a$ for a given $\Delta_{S A S}$ until no mean-field solutions with finite phase winding exist; we discuss a more systematic but numerically more cumbersome approach below. The result of this calculation is summarized in Fig. 12 in which the regime of stable staggered-current-carrying soliton lattice states is plotted as a function of $\Delta_{S A S}$ and the soliton spacing $a$ for $d=\ell$. For $\Delta_{S A S} \rightarrow 0$, the soliton width diverges and the phase $\varphi(X)$ is described by a plane-wave with wavevector $Q$. The phase diagram that we calculate here corresponds in this limit to the $K_{\varphi \varphi}$ stability criterion and the maximum wavevector $Q_{*}$, agrees with that found earlier (see Figs.4,6) to within numerical accuracy.

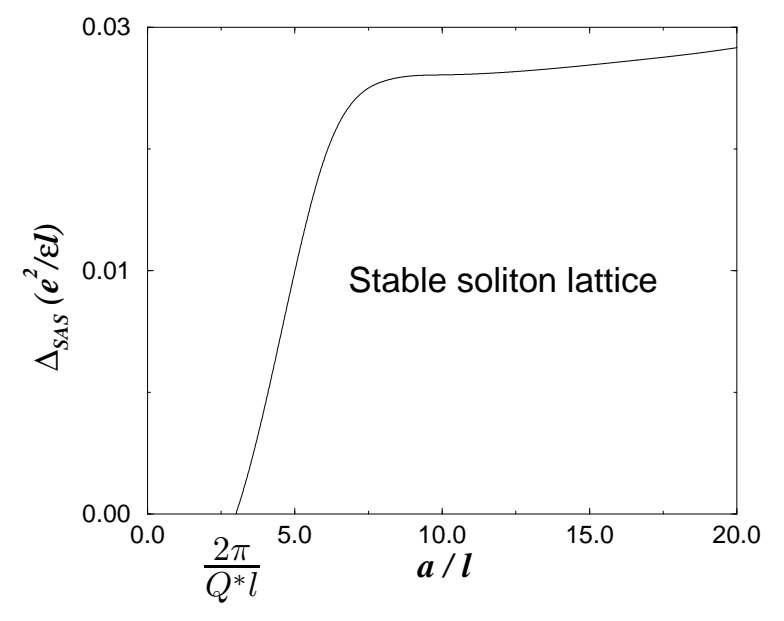

FIG. 12. The stability region for soliton-lattice states, plotted as a function of $\Delta_{S A S}$ and soliton spacing $a$ for $d=\ell$. This phase diagram is constructed by solving the mean-field equations. Metastability in the $\eta=0$ plane holds in the region labeled Stable Soliton Lattice.

This phase boundary shape can be summarized by saying that there is both a minimum distance between solitons and a maximum interlayer tunneling amplitude at which metastable extrema of the energy functional exist. The physics behind this result is not difficult to understand. For weak tunneling, the phase gradient, $\partial_{X} \varphi \approx 2 \pi / \xi \propto \sqrt{\Delta_{S A S}}$ of an isolated soliton $(a \gg \xi)$ is below the critical value of $Q_{*}$ plotted as a dashed line in the inset of Fig. 6. In this regime the stability of the soliton state is therefore predominantly controlled by the soliton spacing $a$, which, for a sufficiently dense soliton lattice leads to phase gradient larger than $Q_{*}$. In contrast, for sufficiently large interlayer tunneling $\Delta_{S A S}$ even an isolated soliton becomes unstable, when its maximum phase gradient $\partial_{X} \varphi \approx 2 \pi / \xi \propto \sqrt{\Delta_{S A S}}$ exceeds $Q_{*}$. Consequently, in the large $\Delta_{S A S}$, large soliton-spacing (a) regime, the phase boundary is not sensitive to soliton spacing $a$, as reflected by the approximately horizontal orientation illustrated in Fig.12. We emphasize that the phase diagram constructed in this way does not account for $K_{z z}$ instabilities.

The soliton lattice state carries a staggered supercurrent which can be evaluated from Eq. 7 given mean-fieldtheory numerical results for the dependence of the energy per unit length perpendicular to the current direction $\varepsilon=8 \rho_{s}^{\perp} / \xi$ on soliton density. (The experimental significance of Eq. 7 is discussed at greater length in Section VI.) For each $\Delta_{S A S}$, the energy per unit length for low soliton density $(a \rightarrow \infty)$ is proportional to the soliton density and the supercurrent therefore approaches a minimum value as $a \rightarrow \infty: J_{c 1}=(4 / \pi) \sqrt{\rho_{s} \Delta_{S A S} / \pi^{3}}$. $[48,19]$ Evidently $J_{c 1}$ decreases by decreasing the interlayer tunneling, and it vanishes like $\Delta_{S A S}^{1 / 2}$ as $\Delta_{S A S} \rightarrow 0$.

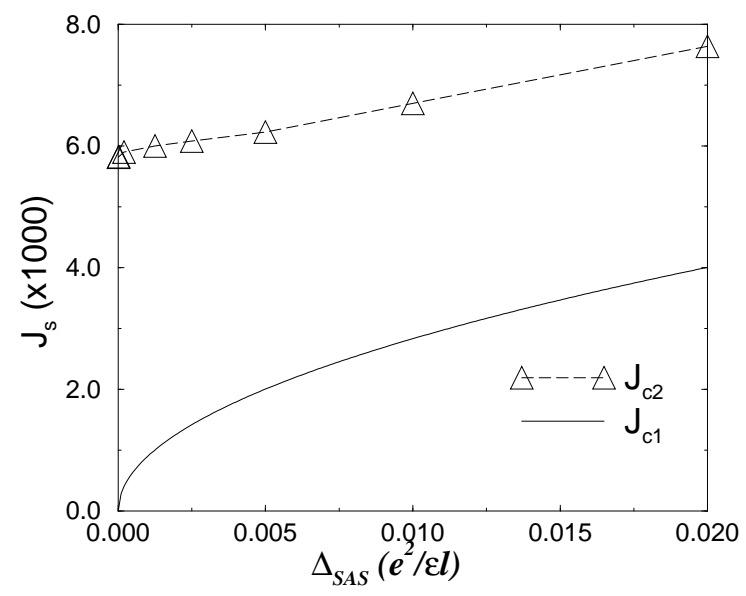

FIG. 13. Maximum and minimum for the persistent current density of soliton lattice states in quantum Hall ferromagnets vs. $\Delta_{S A S}$. $J_{c 1} \propto \sqrt{\Delta_{S A S}}$ for $\Delta_{S A S} \rightarrow 0$.

At large staggered current we also find a maximum sustainable supercurrent density $J_{c 2}$, that corresponds to a large soliton density. In this dense regime, defined by $\xi / a \gg 1$ solitons overlap and current is nearly uni- 
form, leading to a highly oscillating tunneling energy that therefore averages away to zero. Consequently, we expect that $J_{c 2}$ to be well-approximated by $J_{*}$ arising from our earlier criteria of $K_{\varphi \varphi}=0$ for $\Delta_{S A S}=0$. Consistent with this, we find that $J_{c 2}$, shown in Fig.13, is not very sensitive to the tunneling matrix element, increasing only slowly with $\Delta_{S A S}$.

The experimental meaning of $J_{c 1}$ is unclear since one might expect to find solutions to generalized sine-Gordon equations for which the spatially averaged staggered supercurrent in a finite length systems has an arbitrarily small value, for example by having only a partial soliton across the entire system. A staggered current imposed at the edge of the system would fix $\partial_{X} \phi_{X} \mid \propto J_{s}$ only near the boundaries of the bilayer. Provided that these boundary conditions are physically realistic, a question that would require further microscopic analysis to verify, extrema of the functional exist for all current values at the edge, and for all spatially averaged staggered currents. We discuss this point again in Section VI.

\section{B. $K_{z z}$ and $K_{\varphi \varphi}$ staggered current instabilities}

A more systematic method to determine whether or not a solution of the mean-field equations is metastable is to calculate the eigenvalues of both matrices $K_{z z}\left(X, X^{\prime}\right)$, and $K_{\varphi \varphi}\left(X, X^{\prime}\right)$, evaluated at the corresponding energy functional extremum. We have made some progress with this approach, although we have found it to be (unsurprisingly) numerically cumbersome. To make the calculation feasible, we take the length $L_{y}$ of the system in the $y$ direction to be finite, thereby discretizing the set of allowed guiding centers along the $\hat{x}$-axis. For $N$ guiding centers per soliton, $K_{z z}\left(X, X^{\prime}\right)$ and $K_{\varphi \varphi}\left(X, X^{\prime}\right)$ are $N \times N$ matrices. In this study we increase $N$ as far as is practical, then attempt to extrapolate to $N=\infty$. Our numerical results are summarized in Figs. (14-17) for a bilayer electron system with $d=\ell$.

In discussing these results, it is instructive to start with $\Delta_{S A S}=0$, since we can compare with the results obtained analytically in previous sections by Fourier transformation. In Fig. 14, we present spectrum of $K_{z z}$, and $K_{\varphi \varphi}$ evaluated at $\Delta_{S A S}=0$ and $d=\ell$. Starting from a large soliton spacing $a$ (low staggered current), we see that the lowest eigenvalue of $K_{z z}$ decreases with decreasing $a$, finally vanishing for $a \sim 5.2 \ell$, consistent with the value of $Q$ at which the $K_{z z}$ instability occurs in Fig.5. Because of the global phase invariance at $\Delta_{S A S}=0$, there should always be one zero eigenvalue of $K_{\varphi \varphi}$ at any value of $a$, an expectation that we verify within numerical accuracy. It appears from Fig.14, that the finite values of $L_{y}$ that we use for the case of inter-soliton distance $a=40 \ell\left(L_{y}=20 \pi \ell\right)$ are sufficiently short that we introduce artificial instabilities that do not occur for
$L_{y} \rightarrow \infty$. For smaller values of $a$, we are able to consider larger values of $L_{y}$ without making our matrices inconveniently large. These calculations were performed with $L_{y}=800 \pi \ell^{2} / a$. For $a=40 \ell$ we have verified that the negative eigenvalue becomes smaller in magnitude, decreasing toward zero as $L_{y}$ is increased. Consistent with the analytical results, we find a large number of higher eigenvalues of $K_{\varphi \varphi}$ approach zero as $a$ approaches $a^{*} \simeq 3.9 \ell$, the value of $a=2 \pi / Q$ at which the phase instability occurs for $\Delta_{S A S}=0$. The smallest magnitude non-zero eigenvalue of $K_{\varphi \varphi}$ becomes negative at $a^{*}=3.9 \ell$, in reasonable agreement with the location of the supercurrent peak in Fig.4.

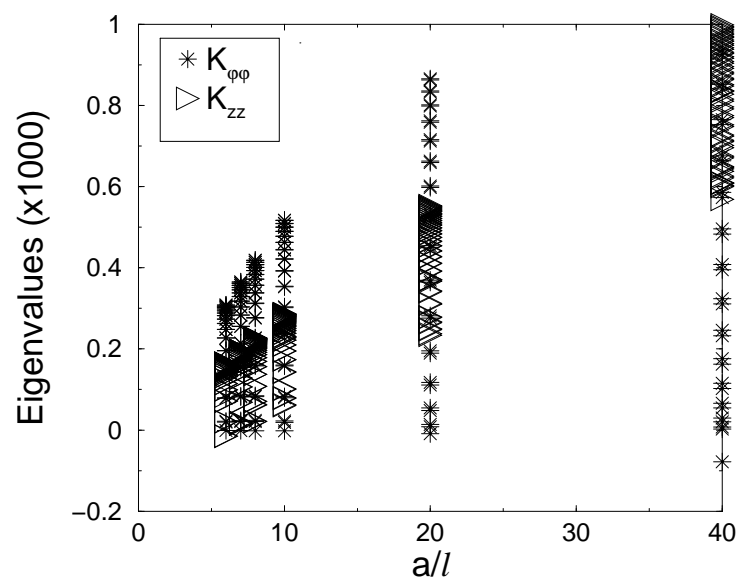

FIG. 14. Stability matrix eigenvalues for $N=400$ and $\Delta_{S A S}=0$ as a function of phase period $a$. The pairing wavevector $Q=2 \pi / a$.

In Fig. 15 we show how the stability matrix spectra depend on $\Delta_{S A S}$. We find that for a given value of $a$, increasing $\Delta_{S A S}$ always leads to an instability. For $K_{\varphi, \varphi}$ this finding is consistent with the mean-field calculation results discussed above. The present results indicate that $K_{z z}$ becomes unstable near the same value of $\Delta_{S A S}$ as $K_{\varphi, \varphi}$; because of the finite values we must use for $L_{y}$ we have not been able to determine which instability occurs first with a high degree of certainty. Figs.15-17, indicate that negative eigenvalues in $K_{z z}$ do appear quite generally when the tunneling energy increases. The instabilities seem to appear first in $K_{z z}$ for small $a$ and first in $K_{\varphi, \varphi}$ for large $a$. At $a=10 \ell$ the smallest eigenvalue of $K_{z z}$ becomes negative for $\Delta_{S A S} \approx 0.028 e^{2} /(\epsilon \ell)$, near where our the mean-field theory $L_{y} \rightarrow \infty$ results indicate the first $K_{\varphi, \varphi}$ instability. Interestingly, these instabilities at finite $\Delta_{S A S}$ have no counterpart in the sine-Gordon theory of the soliton lattice state. They can be understood as being analogous to the maximum currents and pairing wavevectors $Q$ that we find in the translationally invariant case. As $\Delta_{S A S}$ increases the maximum rate of phase winding at the center of a soli- 
ton, $Q_{e f f}=\partial \varphi / \partial X$, increases. For an isolated soliton in the sine-Gordon model $Q_{e f f} \approx 2 \pi / \xi \propto \sqrt{\Delta_{S A S}}$. Thus a maximum tunneling amplitude $\Delta_{S A S} \sim 0.3 e^{2} / \epsilon \ell$ is not unexpected given our results for the homogeneous case.

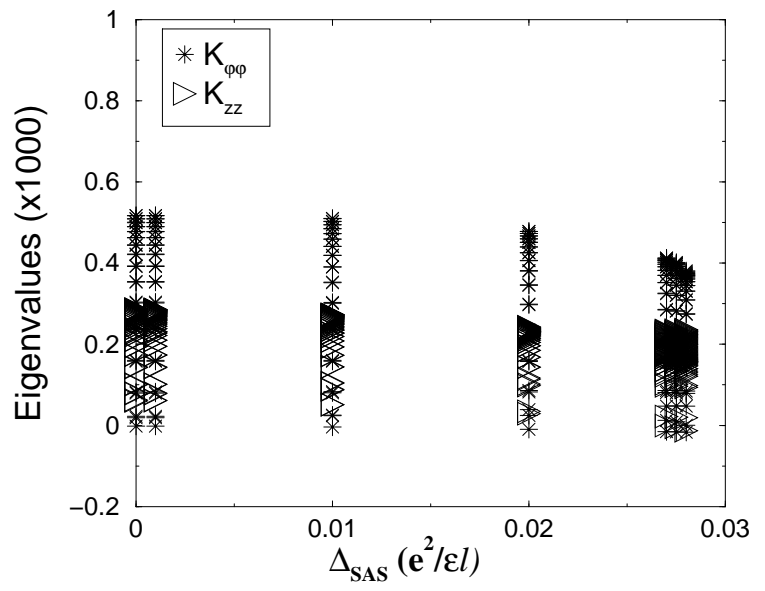

FIG. 15. Stability matrix eigenvalues for $N=400$ and $a=10 \ell$ as a function of $\Delta_{S A S}$.

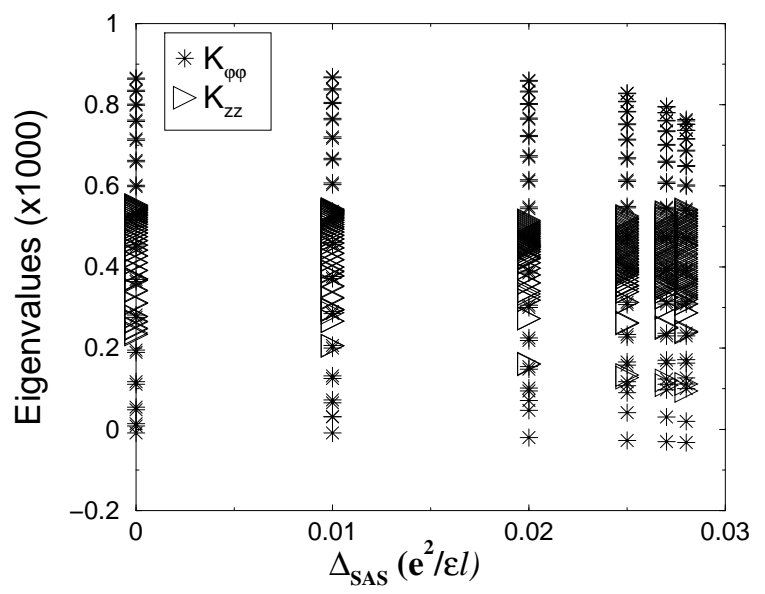

FIG. 16. Stability matrix eigenvalues for $N=400$ and $a=20 \ell$ as a function of $\Delta_{S A S}$.

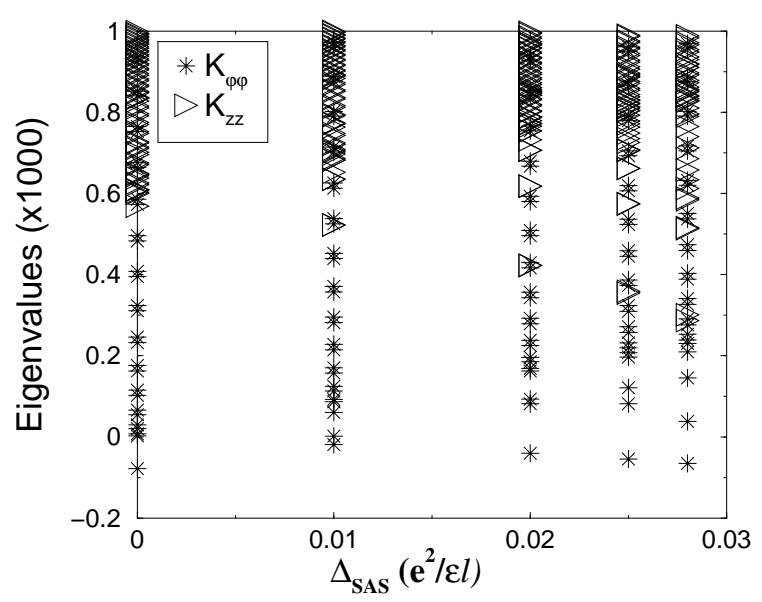

FIG. 17. Stability matrix eigenvalues for $N=400$, and $a=40 \ell$ as a function of $\Delta_{S A S}$.

\section{DISCUSSION AND CONCLUSIONS}

In this paper we have presented a mean-field theory of staggered supercurrent carrying states in quantum Hall bilayer superfluids. Our analysis was based primarily on the behavior of two stiffnesses $K_{z z}$ and $K_{\varphi \varphi}$ that characterize respectively the energy cost of small interlayer charge and interlayer phase fluctuations. By computing these quantities, we have been able to address the physics that controls the dependence of critical staggered current on interlayer tunneling amplitude, layer spacing, and other tunable parameters that characterize bilayer systems. We also studied the characteristics of weak links in quantum Hall superfluids that are created by a gate voltage. Unlike the field-theoretic approach [19], the microscopic analysis here does not rely on a small wavevector expansion, and therefore has the virtue of accounting for the full wavevector dependence of the stability matrices. This is especially important because the wavevector expansion of these matrices is not analytic which indirectly causes these quantities to have minima at finite wavevectors. The staggered critical supercurrent predictions presented in this paper are consequently more realistic than those derived from the field-theoretic approach [19]. 


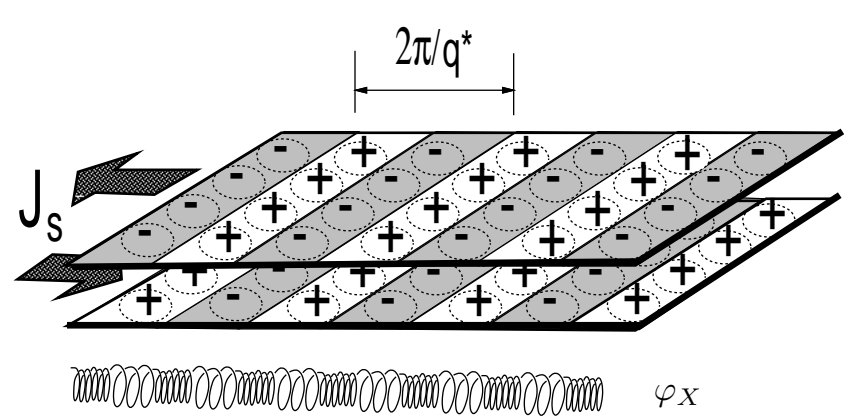

FIG. 18. Schematic representation of the staggered current-induced QH supersolid state for which periodic interlayer charge modulation and QH superfluidity coexist. The periodic rate of phase winding is also illustrated.

There are a number of limitations to the approach we have followed. Our analysis is confined to harmonic instabilities and will therefore miss any that are induced through a nonlinear mode, nucleation of vortices for example. In addition, our harmonic analysis does not unambiguously determine the state to which the system evolves when the instability point is reached. Based on experience with ordinary superfluids and superconductors, a natural guess is that once one of the stiffnesses vanishes, the current-carrying superfluid state of the quantum Hall bilayer is unstable to a resistive (normal) state in which staggered current leads to a staggered voltage. We believe that this scenario applies when $K_{\varphi \varphi}$ controls the instability, and when the instability in $K_{z z}(q)$ takes place at a large wavevector corresponding to a length scale of order or smaller than the magnetic length $\ell$. The instability process has that character for the large interlayer spacing values, $d \gtrsim \ell$, relevant to current experiments. However, in the opposite limit, $d<<\ell$, it is likely (based on similarity with our earlier work on the interlayer-charge imbalance instability driven by an in-plane magnetic field $[50,37])$ that vanishing $K_{z z}\left(q^{*}\right)$ instead signals an interlayer charge imbalance quantum (nonequilibrium) transition to a distinct staggered current carrying QH superfluid state. As with the equilibrium canting transition $[50,37]$ at small $d / \ell$, the instability signaled by vanishing of $K_{z z}\left(q^{*}\right)$ occurs at a small but finite wavevector $q^{*}$ (with $q^{*} \ell<<1$ ), and therefore cor- responds to a development of a dipole-stripe state akin to a unidirectional charge density state with period $2 \pi / q^{*}$, as illustrated in Fig.18. Since, in addition to staggered gauge symmetry, such a state also spontaneous breaks translational invariance along the staggered current direction, it corresponds to a quantum Hall supersolid with coexisting superfluid, solid, and quantum Hall orders.

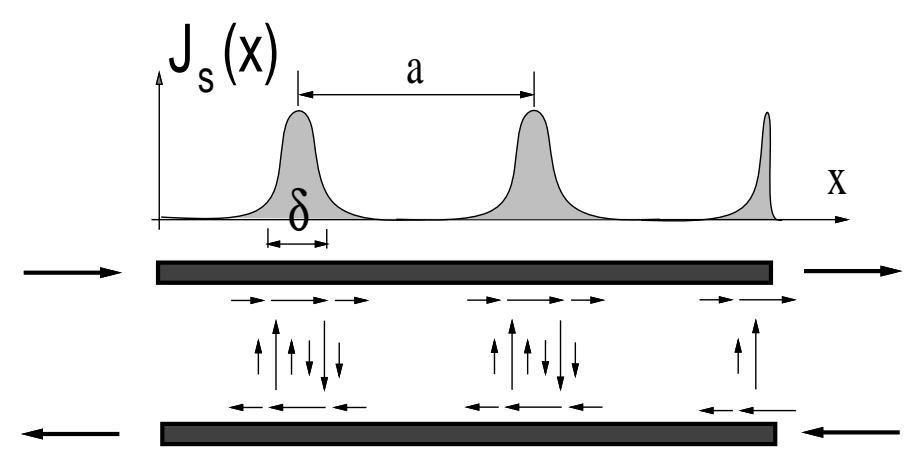

FIG. 19. Schematic QH bilayer in the presence of finite single-particle interlayer tunneling $\Delta_{S A S}$. When there is collective tunneling between the layers, the staggered supercurrent must be spatially dependent in order to conserve the difference in charge between the layers. The spatially averaged staggered supercurrent can be arbitrarily small in a finite systems because of edge effects. The soliton width in this illustration $\delta=\ell \sqrt{\rho_{s} / \Delta_{S A S}}$, while the period $a$ is the distance between soliton centers. This schematic plot shows a partial soliton on the right that can make an arbitrarily small contribution to the spatially averaged staggered supercurrent.

Another feature of our work is the finding that the staggered super current evaluated for soliton-lattice states using Eq. 7 has a minimum value $J_{c 1}$ as well as a maximum value. This expression for the staggered current can be motivated in several different ways, but its connection to experiment is not always direct, as we now explain. The general expression for the staggered current can be derived by introducing a spatially constant staggered vector potential $A_{\uparrow}=-A_{\downarrow}=A / 2$ and noting that the interaction energy is then a function of the gauge invariant inter-layer phase difference $\varphi_{X}+e X A / \hbar c$. It follows that the staggered current is given by

$$
\begin{aligned}
J_{s} & =\frac{1}{\hbar L_{x} L_{y}} \frac{\partial E}{\partial A_{\uparrow}} \\
& =\frac{1}{2 \hbar L_{x} L_{y}^{2}} \sum_{X, X^{\prime}} F_{D}\left(X-X^{\prime}\right) \sin \theta(X) \sin \theta\left(X^{\prime}\right) \sin \left[\varphi(X)-\varphi\left(X^{\prime}\right)+e\left(X-X^{\prime}\right) A / \hbar c\right]\left(X-X^{\prime}\right) .
\end{aligned}
$$

The vector potential $A$ has no physical meaning if $\Delta_{S A S}=0$. For $\Delta_{S A S} \neq 0$, however, an in-plane field is represented by $A=B_{\|} d$ and in equilibrium Eq. 14 de- scribes the persistent currents responsible for orbital diamagnetism. In the absence of an in-plane field $A=0$, and $\varphi(X)$ is determined by minimizing Eq. 2 . When the mini- 
mization procedure is carried out with periodic boundary conditions, as in our numerical calculations, changing $A$ is equivalent to changing the period over which $\varphi$ is required to change by $2 \pi$, and Eq. 7 follows. For realistic systems, however, edge effects can have a large effect on the current, especially when individual solitons, which carry circulating currents in their cores, are well separated.

$J_{c 1}$ is analogous to the lower critical magnetic field $H_{c 1}$ in type-II superconductors. Only for $J_{s}>J_{c 1}$ does it pay for the system to give up some of the tunneling kinetic energy and allow a soliton that carries this minimum staggered current $J_{c 1}$ into the system. $J_{c 1}$ is closely related to the critical in-plane magnetic field that induces the commensurate-incommensurate transitions in quantum Hall bilayers. $[9,50]$

Consider, for example, the limit of slowly varying phases for which the local staggered current density can be shown to be [9] $2 \rho(\partial \varphi / \partial x)$, in agreement with Eq. 14. In this case the spatially averaged staggered current is proportioanl to the number of solitons in the system, which can clearly be arbitrarily small. This conclusion should be contrasted with that reached by Shevchenko [48], working with a closely related longwavelength model that applies to the case of of electronhole bilayers at zero magnetic field. (Excitonic superfluidity has not yet been conclusively established in experiment for this case.) Shevchenko's conclusion that $J_{c 1}$ would represent an experimental minimum staggered supercurrent was based on the argument that finite current configurations could be studied by adding a soliton chemical potential term [more precisely a "chemical potential" for interlayer phase twist] $J_{s} 2 \rho_{s} \partial_{X} \varphi / \hbar$ to the bilayer energy density functional. This term is chosen so that the modified energy functional has its absolute minimum when Eq. 7 for the current density is satisfied, and is modelled after related approximations that are common [49] in the description of current-biased Josephson junctions. In this approach, because for finite tunneling $\Delta_{S A S}$ the induced phase twist costs interlayer kinetic energy (tunneling), a minimum staggered current $J_{c 1}$ is required for phase winding to occur. Below this critical value, $J_{s}<J_{c 1}$ (analogous to a Meissner state of typeII superconductor, where vortex density $\propto B$ vanishes) no solitons are induced, and phase $\varphi_{X}$ remains spatially uniform and pinned at 0 (except for the boundaries). The consequent vanishing of the interlayer tunneling current leads to a conserved staggered current $J_{s}(x)$, that is therefore spatially uniform. In our view, however, this thermodynamic description of finite current states does not apply to electron-hole bilayer systems with excitonic superfluidity, either at zero field or in the quantum Hall regime. The key difference between quantum Hall bilayers and Josephson junctions is the absence of a superconducting current-bias environment which imposes a definite value of the staggered curent at every point in space.
In the bilayer quantum Hall case, current-carrying states are never equilibrium states, but they can be metastable. External contacts can in principle, impose a given value of the staggered current at every point on the edge of the system, assuming that non-collective current carrying excitations can be completely discounted. However, the staggered current density at interior points must be determined by finding local minima of the energy functional that are consistent with edge boundary conditions. In general when $\Delta_{S A S}$ is non-zero, many such solutions exist. The critical current is naturally defined as the metastable configuration with the largest staggered supercurrent, even for geometries that are more general than those considered here.

It is difficult for several reasons to use our microscopic HFA approach to calculate the activation energies for the saddle point extrema that separate local minima of the HF energy with different numbers of phase slips. These activation energies will control the thermal phase-slip nucleation rate and the associated staggered voltage at any finite temperature. One problem encountered in seeking these solutions is that standard simple iterative approaches can be used to find solutions of the mean-field equations that correspond to local minima, whereas some more subtle and clever technique would have to be used to coax the system iteratively toward a saddle-point solution. A second problem is that for the wide Hall bars of interest to us here, the saddle point solution likely consists of nucleating a vortex and moving it to the middle of the Hall bar; these solutions have polar and azimuthal angle fields that depend on both spatial coordinates, making the problem two-dimensional.

We can, however, make some progress toward understanding staggered channel dissipation by combining our HFA results and a scaling analysis supported by exact field-theoretic calculations valid for a short-range interaction model [19]. For example, consider the case of thermally-activated staggered resistance controlled by a saddle-point phase-slip solution $m_{z}^{s p}(x)$, similar to the one illustrated in Fig.20. We expect that the limiting saddle point will have this character for narrow Hall bars. The phase slip is characterized by a single length scale

$$
\xi=\frac{\xi_{0}}{\sqrt{1-\xi_{0}^{2} q_{*}^{2}}}
$$

which reduces to the width $\xi_{0}=\sqrt{\rho_{s} / K_{z z}(0)}$ [19] of saddle-point solution in the limit of short-range interactions or small $\xi_{0} q_{*}$, valid for $d / \ell \ll 1 ; q_{*}$ is the wavevector at which, in the presence of long-range Coulomb interaction $K_{z z}(q)$ is minimum. In this short-range limit, we expect the saddle-point solution $m_{z}^{s p}(x)$ and the associated energy barrier to be well described by the field-theoretic prediction [19]. 


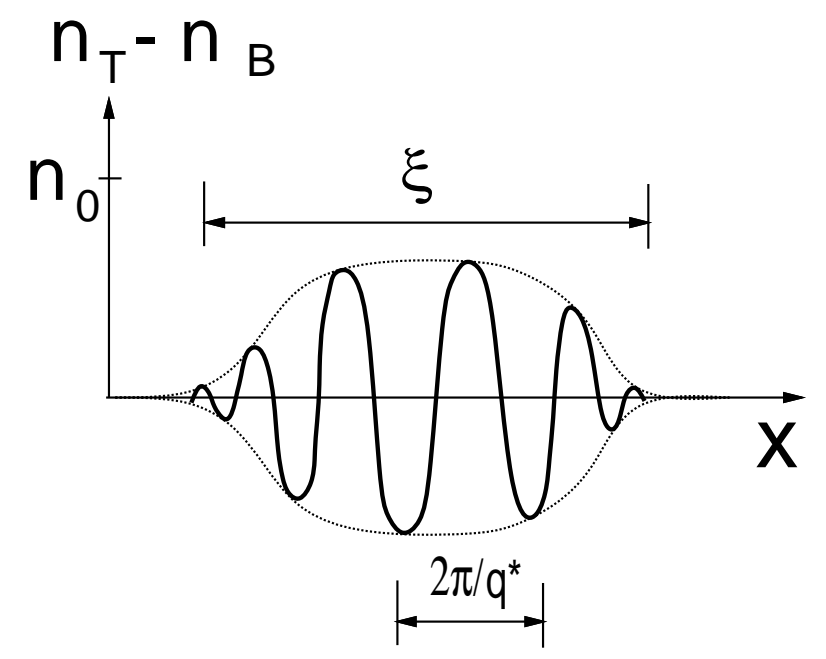

FIG. 20. Schematic illustration of the saddle-point solution, $m_{z}^{s p}(x)$ for $\xi_{0} q_{*} \rightarrow 1^{-}$, characterized by envelope width $\xi$ and wavevector $q_{*}$. The free-energy associated with this $m_{z}^{s p}(x)$ controls the energy barriers for thermal activation of phase slips and dissipation in the staggered current channel when the Hall bar is narrow.

In the opposite limit, as the width $\xi_{0}$ of the soliton nucleation site approaches (from below) the period $2 \pi / q_{*}$ at which the interlayer charge-imbalance $m_{z}=n_{T}-n_{B}$ instability takes place, we expect a saddle-point solution to be qualitatively modified by long-range Coulomb interactions. In this regime we predict an oscillatory $m_{z}^{s p}(x)$, with wavevector $q_{*}$ and an envelope of width $\xi$, as illustrated in Fig.20. The associated energy barrier in the limit of vanishing staggered current is given by

$$
E_{B}=\epsilon_{B}^{0}\left(1-\xi_{0}^{2} q_{*}^{2}\right)^{1 / 2} L_{y}
$$

where $\epsilon_{B}^{0}$ the energy barrier per unit of soliton length in the limit of short-range interactions [19].

This analysis has been limited so far to onedimensional instabilities. The energy barrier associated with such a one-dimensional soliton grows with the width $L_{y}$ of the quantum Hall bar. In contrast, for vanishing tunneling $\Delta_{S A S}=0$, barriers for point-vortex nucleation are proportional to $\rho_{s}$, up to corrections logarithmic in $L_{y}$. Consequently, for sufficiently wide quantum Hall bars, $L_{y}>\xi[19]$, the staggered resistivity will be determined by point-vortex nucleation and unbinding. Indeed standard arguments guarantee that some dissipation will always be present at finite temperatures and voltages, as we discuss below.

Our discussion has so far ignored quantum and thermal fluctuations and quenched disorder, present in real systems. For layer separations $\sim 10 \%$ below the critical value $d_{c}[52,53]$, quantum fluctuations in the absence of quenched disorder can be treated perturbatively [52] and will yield only small corrections to our results. In contrast, thermal fluctuations and especially in combina- tion with disorder can modify some of our results qualitatively. Even in the absence of quenched disorder, for vanishing tunneling, thermal fluctuations lead to a finite, activated dissipation (i.e., staggered voltage) at any finite staggered current, and therefore as in the case of superconductors, preclude an unambiguous definition of a critical current. In this case a superfluid state is distinguished from a dissipative one by a vanishing linear resistivity. Extending standard arguments [51] to our system for $\Delta_{S A S}=0$ limit, we predict a nonlinear power-law staggered I-V characteristic $E_{s} \sim J_{s}^{\alpha}, \alpha(T) \geq 3$ to characterize the quantum Hall staggered superfluid transport. Despite finite dissipation even below mean-field critical staggered current $J_{c}$ computed here, at low temperatures we nevertheless expect a strong crossover in the I- $\mathrm{V}$ at $J_{c}$, with the staggered voltage drastically dropping for current below $J_{c}$ and dissipation controlled by thermally activated vortex nucleation and vortex transport over barriers.
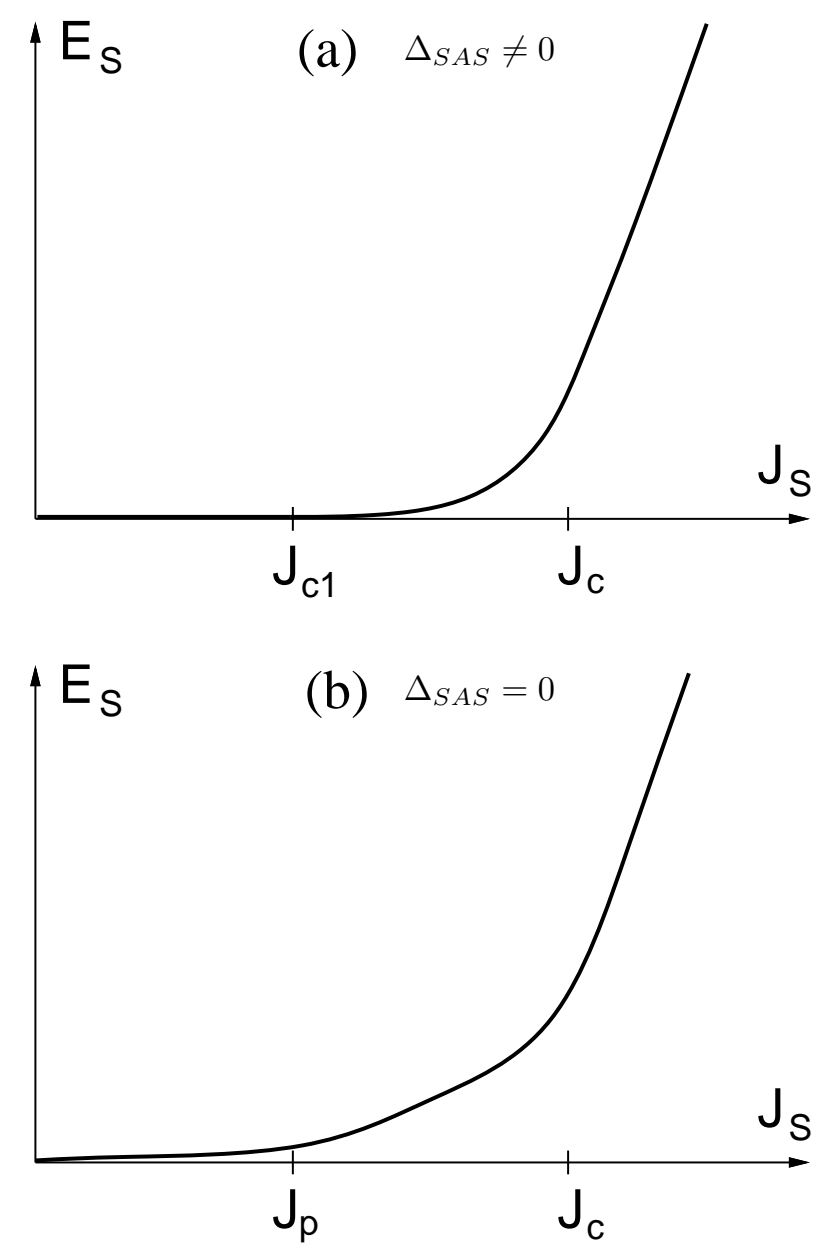

FIG. 21. Low temperature and weak disorder staggered current-voltage (I-V) characteristics for QH bilayers for (a) finite and (b) vanishing interlayer tunneling $\Delta_{S A S}$. 
For finite interlayer tunneling, $\Delta_{S A S} \neq 0$, the ionization energy necessary to separate vortex pairs grows linearly with separation $R$ as $\left(\rho_{s} \Delta_{S A S}\right)^{1 / 2} R / \ell$. Consequently, in thermodynamic limit (wide bilayers) we should expect a true staggered critical current $J_{c 1}=$ $(1 / \hbar \ell)\left(\rho_{s} \Delta_{S A S}\right)^{1 / 2}$, below which staggered transport is dissipationless, $E\left(J_{s}<J_{c 1}\right)=0$, even at finite temperature, and for $J_{s}>J_{c 1}, E(J) \propto\left|J_{s}-J_{c 1}\right|^{\alpha(T)}$ [19]. The corresponding I-V is illustrated in Fig.21a.

Quenched disorder is another important ingredient that we have not considered in detail here. As we have already emphasized, our analysis of the weak-link strongly suggests that by creating weak links, disorder will suppress critical currents. Short-range disorder potential fluctuations will tend to nucleate vortices, a fact that can be understood in terms of the short-range-correlated random effective vector potential $[54,25]$ that it gives rise to. For $\Delta_{S A S} \rightarrow 0$, the random vector potential model is known to have a stable low temperature quasi-long-range ordered phase with no unbound vortices that appears below a critical strength of disorder. [55] (Microscopic calculations [52] that treat disorder using a self-consistent Born approximation, lead to the same conclusion.) Beyond the presumed disorder threshold, the system is in the gauge-glass regime, which in two dimensions is unstable to vortices that are mobile at finite temperature and only localize as $T \rightarrow 0$. Hence in the weak disorder limit, when vortices are dilute, $\delta \ll L_{L}$ (where $L_{L}$ is the Larkin length), the mean-field critical current found in this paper should be clearly observable. In fact for weak disorder we expect to see three regimes of dissipation via motion of disorder-induced vortices: (i) slow, thermally activated creep for $J_{s}<J_{p}$, (ii) vortex flow, for $J_{p}<J_{s}<J_{c}$ (where $J_{c}$ is the mean-field critical current of a clean system derived here), and (iii) normal state dissipation for $J_{c}<J_{s}$, when the system is past its point of metastability. The corresponding I-V is schematically illustrated in Fig.21b.

How then might the critical currents calculated here, which are based on a Landau-like metastability requirement for the staggered-current carrying state, appear experimentally? The best possibility appears to be by looking for features in the non-linear I-V relationships seen in interlayer quantum Hall drag experiments. [23] So far these experiments seem to show a linear relationship between the longitudinal staggered voltage and the staggered current, with a thermally activated dissipation process. There is no hint at present that the staggered electric field initially grows sublinearly with current, suggesting that $J_{p}$ might be driven to zero by disorder. It will be very interesting to see whether or not a relatively sharp crossover can be observed experimentally at low temperatures at a current density that can be associated with $J_{c}$. The crossover would be from a regime in which the staggered current is largely collective, but not completely dissipationless due to the motion of vortices nu- cleated thermally and by disorder, to a regime in which there is essentially no collective staggered current. If our estimates are qualitatively reliable, the scale of $J_{c}$ is normally larger than those used in typical experiments but vanishes as $\left(d_{c}-d\right)^{1 / 2}$ as the phase boundary for the spontaneous coherence state is approached from the below. For this reason, we believe that nonlinear transport experiments in the drag geometry, current in one layer and voltages measured in both layers, as a function of $d_{c}-d$ will be helpful in sorting out the complex physics present in current samples. [23]

\section{ACKNOWLEDGEMENTS}

We gratefully acknowledge helpful interactions with Leon Balents, Anton Burkov, Herb Fertig, Steve Girvin, Bert Halperin, Yogesh Joglekar, Jordan Kyriakidis, Enrico Rossi, John Schliemann, and Ady Stern. This work was supported by the Welch Foundation, by the National Research Council under grants DMR-0115947 and NSF MRSEC DMR-0080054, and by the Sloan and Packard Foundations.

[1] B.I. Halperin, Helv. Phys. Acta 56, 75 (1983).

[2] M. Rasolt and A.H. MacDonald, Phys. Rev B 34, 5530 (1986).

[3] H. Fertig, Phys. Rev. B 40, 1087 (1989).

[4] Daijiro Yoshioka and A.H. MacDonald, J. Phys. Soc. Jpn. 59, 4211 (1990); A. H. MacDonald and E.H. Rezayi, Phys. Rev. B 42, 3224 (1990); A.H. MacDonald, E.H. Rezayi, and D. Keller, Phys. Rev. Lett. 68, 1939 (1992).

[5] A. H. MacDonald, P.M. Platzman, and G.S. Boebinger, Phys. Rev. Lett. 65, 775 (1990).

[6] For a review see S.M. Girvin and A.H. MacDonald in Perspectives in Quantum Hall Effects, edited by S. Das Sarma and Aron Pinczuk (Wiley, New York, 1997).

[7] X.G. Wen and A. Zee, Phys. Rev. Lett. 69, 1811 (1992).

[8] Z. F. Ezawa and A. Iwazaki, Int. J. Mod. Phys. B 6, 3205 (1992); Phys. Rev. B 47, 2265 (1993).

[9] K. Moon, H. Mori, Kun Yang, S.M. Girvin, and A.H. MacDonald, L. Zheng, D. Yoshioka, and Shou-cheng Zhang, Phys. Rev. B 51, 5138 (1995); Kun Yang, K. Moon, Lotfi Belkhir, H. Mori, S.M. Girvin, A.H. MacDonald, L. Zheng, and D. Yoshioka, Phys. Rev. B 54, 11644 (1996).

[10] S. Q. Murphy, J. P. Eisenstein, G. S. Boebinger, L. N. Pfeiffer, K. W. West, Phys. Rev. Lett. 72, 728 (1994).

[11] L.V. Keldysh and Yu. V. Kopaev, Sov. Phys. Solid State 6, 2219 (1965); J. des Cloizeaux, J. Phys. Chem. Solids 26, 259 (1965); B.I. Halperin and T.M. Rice, Solid State Physics, 21, F. Seitz, D. Turnbull and H. Ehrenreich, eds., Academic Press, New York, 1968. 
[12] L.V. Butov et al., Nature 41747 (2002); D.W. Snoke et al. Int. J. Mod. Phys. 15, 3606 (2001); L.V. Butov et al., Nature 418, 751 (2002); D. Snoke et al., Nature 418, 754 (2002).

[13] D.P. Young, D. Hall, M.E. Torelli, Z. Fisk, J.L. Sarao, J.D. Thompson, H.-R. Ott, S. B. Osheroff, R.G. Goodrich and R. Zysler, Nature 397, 412 (1999).

[14] M.E. Zhitomirsky, T.M. Rice and V.I. Anisimov, Nature 402, 251 (1999); Leni Bascones, Anton Burkov and A.H. MacDonald, Phys. Rev. Lett. 89, 086401 (2002).

[15] M. R. Matthews, B. P. Anderson, P. C. Haljan, D. S. Hall, M. J. Holland, J. E. Williams, C. E. Wieman, and E. A. Cornell, Phys. Rev. Lett. 83, 3358 (1999).

[16] Yu. E. Lozovik and V.I. Yudson, Pis'ma Zh. Eksp. Teor. Fiz. 22556 (1975) [JETP Lett. 22, 271 (1975)]; ibid, Zh. Eksp. Teor. Fiz. 71, 738 (1976) [Sov. Phys. JETP 44, 389 (1976)]; S.I. Shevchenko, Fiz. Nizk. Temp. 2, 505 (1976) [Sov. J. Low Temp. Phys. 2, 251 (1976)]; X. Zhu, P.B. Littlewood, M. Hybertsen, and T.M. Rice, Phys. Rev. Lett. 74, 1633 (1995).

[17] Y. Kuramoto and C. Horie, Solid State Commun. 25, 713 (1979); I.V. Lerner, and Yu. E. Lozovik, Zh. Eksp. Teor. Fiz. 80, 1488 (1981) [Sov. Phys. JETP 53, 763 (1981)]; Y.A. Bychkov and E.I. Rashba, Solid State Commun. 48, 399 (1983).

[18] D. Paquet, T.M. Rice, and K. Ueda, Phys. Rev. B 32, 5208 (1985). Note that this paper incorrectly concludes that the interaction between excitons is attractive, implying droplet formation rather than a uniform superfluid state. In the pseudospin ferromagnet language, this conclusion corresponds to predicted easy-axis, rather than easy-plane order.

[19] J. Kyriakidis and L. Radzihovsky, Phys. Rev. B 64, 201314 (2001).

[20] For a brief review see J.P Eisenstein, M.P. Lilly, K.B. Cooper, L.N. Pfeiffer, K.W. West, Proceedings of the 11th International Winter School on New Developments in Solid State Physics, Mauterndorf, Austria, February, 2000. [to be published in Physica E].

[21] I.B. Spielman, J.P. Eisenstein, L.N. Pfeiffer, and K.W. West, Phys. Rev. Lett. 84, 5808 (2000).

[22] I. B. Spielman, J. P. Eisenstein, L. N. Pfeiffer, and K. W. West, Phys. Rev. Lett. 87, 036803 (2001).

[23] M. Kellogg, I.B. Spielman, J.P. Eisenstein, L.N. Pfeiffer, and K.W. West, Phys. Rev. Lett. 88, 126804 (2002).

[24] X.G. Wen and A. Zee, Phys. Rev. B 47, 2265 (1993).

[25] L. Balents, L. Radzihovsky, Phys. Rev. Lett. 86, 1825 (2001); A. Stern et al., ibid. 1829 (2001); M. Fogler and F. Wilczek, ibid. 1833 (2001).

[26] Y. Joglekar and A.H. MacDonald, Phys. Rev. Lett. 87, 196802 (2001).

[27] M. Abolfath, R. Khomeriki, K. Mullen, condmat/0208236.

[28] X.M. Chen and J.J. Quinn, Phys. Rev. Lett. 67, 895 (1991); R. Côté, L. Brey, and A.H. MacDonald, Phys. Rev. B 46, 10239 (1992).

[29] Jürgen König, Martin Chr. Bnsager and A. H. MacDonald Phys. Rev. Lett. 87, 187202 (2001); F.S. Nogueira and K.-H. Bennemann, cond-mat/0302528; Jan Heurich, Jürgen König, and A.H. MacDonald, cond-mat/0304xxx.

[30] B.I. Halperin, Phys. Rev. B 25, 2185 (1982). A.H. Mac-
Donald and P. Streda, Phys. Rev. B 29, 1616 (1984).

[31] A.H. MacDonald, Physica B 298129 (2001).

[32] Giovanni Vignale and A.H. MacDonald, Phys. Rev. Lett. 76, 2786 (1996).

[33] K. Yang, Phys. Rev. Lett. 87, 056802 (2001).

[34] S. M. Girvin, Phys. Scripta T102, 112 (2002).

[35] A.A. Burkov, Y. Joglekar, E. Rossi, and A.H. MacDonald, to be submitted to Phys. Rev. B.

[36] B.I. Halperin and A. Stern, Phys. Rev. Lett. 88, 106801 (2002).

[37] M. Abolfath, L. Radzihovsky and A.H. MacDonald, Phys. Rev. B 65, 233306 (2002).

[38] X.G. Wen, and A. Zee, Europhys. Lett. 35, 227 (1996).

[39] A.A. Burkov and A.H. MacDonald, Phys. Rev. B 66, 115320 (2002).

[40] R. Côté, L. Brey, H. Fertig, and A.H. MacDonald, Phys. Rev. B 51, 13475 (1995).

[41] J. Kyriakidis, D. Loss, and A.H. MacDonald, Phys. Rev. Lett. 83, 1411 (1999).

[42] A.H. MacDonald and Shou-Cheng Zhang, Phys. Rev. B 49, 17208 (1994).

[43] J. S. Langer, and Vinay Ambegaokar, Phys. Rev. 164, 498 (1967).

[44] V. Ambegaokar, and A. Baratoff, Phys. Rev. Lett. 10, 486 (1963).

[45] M. Abolfath, Ramin Golestanian, and T. Jungwirth, Phys. Rev. B 61, 4762 (2000).

[46] C.N. Yang and C. P. Yang, Phys. Rev. 150, 321 (1966).

[47] Here we consider a weak link that is localized in $x$, translationally invariant in $y$ direction and is created capacitively with a top narrow gate that suppresses $\rho_{s}$ via interlayer charge imbalance (see Fig.8). However, other weak links are possible while maintaining interlayer charge balance. One such link can be induced by two narrow side gates that also constrict the bilayer along the $y$ direction. The other possibility is via a narrow bilayer region, where $d / \ell$ ratio has been increased to be near a critical value determined by superfluid state stability. However, this latter configuration might be difficult to realize experimentally.

[48] S. I. Shevchenko, Phys. Rev. Lett. 72, 3242 (1994); Phys. Rev. B 57, 14809 (1998).

[49] See for example Konstantin K. Likharev, Dynamics of Josephson Junctions and Circuits, (Gordon and Breach, Amsterdam, 1986).

[50] L. Radzihovsky, Phys. Rev. Lett. 87, 236802 (2001).

[51] V. Ambegaokar, B. I. Halperin, D. R. Nelson and E. D. Siggia, Phys. Rev. Lett. 40, 783 (1978).

[52] Y.N. Joglekar and A.H. MacDonald, Phys. Rev B 64, 155315 (2001).

[53] Interesting insights into some quantum fluctuation effects can also be extracted from exact diagonalization calculations. See for example J. Schliemann, Phys. Rev. B 67, 025238 (2003); J. Schliemann, S.M. Girvin and A.H. MacDonald, Phys. Rev. Lett. 86, 1849 (2001).

[54] A. Stern, S. Das Sarma, M. P.A. Fisher, and S.M. Girvin, Phys. Rev. Lett. 84, 139 (2000).

[55] T. Nattermann, et al., J. de Physique I 5, 565 (1995).

[56] G. Blatter, M .V. Feigelman, V. B. Geshkenbein, A. I. Larkin, and V. M. Vinokur, Rev. Mod. Phys., 66, 1125 (1994). 